\title{
665.
}

\section{A MEMOIR ON THE DOUBLE 9-FUNCTIONS.}

[From the Journal für die reine und angewandte Mathematik (Crelle), t. Lxxxv. (1878), pp. 214-245.]

I RESUME my investigations on these functions; see my two papers, Crelle, t. LXXXIII. (1877), pp. 210-233; [662] and [663]. But it is proper in the first instance to develope in a corresponding manner, the theories of the circular (or exponential) functions, and of the single 9 -functions.

\section{Part I. Preliminary investigations.}

Starting from the differential relation

$$
\partial u=\frac{\partial x}{\sqrt{a-x \cdot b-x}}
$$

between the variables $u$ and $x, I$ write for shortness the single letters $A, B, \Omega$, instead of functional forms $A(u), B(u), \Omega(u)$, to denote functions of $u$; and I assume as definitions the equations

$$
\begin{aligned}
& A=\Omega \sqrt{a-x}, \\
& B=\Omega \sqrt{b-x},
\end{aligned}
$$

and another equation to be presently mentioned: these two equations imply between $A, B, \Omega$ the algebraical relation

$$
A^{2}-B^{2}=\Omega^{2}(a-b)
$$

Differentiating, we obtain

$$
\partial A=\partial \Omega \cdot \sqrt{a-x}-\frac{\Omega}{2 \sqrt{a-x}} \sqrt{a-x \cdot b-x} \partial u,
$$


that is,

$$
\partial A=\frac{A}{\Omega} \partial \Omega-\frac{1}{2} B \partial u
$$

and similarly

$$
\partial B=\frac{B}{\Omega} \partial \Omega-\frac{1}{2} A \partial u
$$

whence

$$
A \partial B-B \partial A=-\frac{1}{2}\left(A^{2}-B^{2}\right) \partial u .
$$

Proceeding to a second differentiation, we find

$$
\begin{aligned}
& \partial^{2} A=\frac{A}{\Omega} \partial^{2} \Omega-\frac{B}{\Omega} \partial \Omega \partial u+\frac{1}{4} A(\partial u)^{2}, \\
& \partial^{2} B=\frac{B}{\Omega} \partial^{2} \Omega-\frac{A}{\Omega} \partial \Omega \partial u+\frac{1}{4} B(\partial u)^{2},
\end{aligned}
$$

and thence

$$
\begin{aligned}
& A \partial^{2} A-(\partial A)^{2}=\frac{A^{2}}{\Omega^{2}}\left\{\Omega \partial^{2} \Omega-(\partial \Omega)^{2}\right\}+\frac{1}{4}\left(A^{2}-B^{2}\right)(\partial u)^{2} \\
& B \partial^{2} B-(\partial B)^{2}=\frac{B^{2}}{\Omega^{2}}\left\{\Omega \partial^{2} \Omega-(\partial \Omega)^{2}\right\}+\frac{1}{4}\left(B^{2}-A^{2}\right)(\partial u)^{2}
\end{aligned}
$$

To simplify these we assume (as the third equation above referred to)

$$
\Omega \partial^{2} \Omega-(\partial \Omega)^{2}=0 .
$$

The last-mentioned two equations then become

$$
\begin{aligned}
& A \partial^{2} A-(\partial A)^{2}=\frac{1}{4}\left(A^{2}-B^{2}\right)(\partial u)^{2}, \\
& B \partial^{2} B-(\partial B)^{2}=\frac{1}{4}\left(B^{2}-A^{2}\right)(\partial u)^{2},
\end{aligned}
$$

which several equations contain the theory of the functions $A, B, \Omega$ : we have as their general integrals

$$
\begin{aligned}
& A=\frac{1}{2} \Lambda e^{\lambda u} \sqrt{a-b}\left\{e^{\frac{1}{2}(u+v)}+e^{-\frac{1}{2}(u+v)}\right\} \\
& B=-\frac{1}{2} \Lambda e^{\lambda u} \sqrt{a-b}\left\{e^{\frac{1}{2}(u+v)}-e^{-\frac{1}{2}(u+v)}\right\} \\
& \Omega=\Lambda e^{\lambda u}
\end{aligned}
$$

where $\Lambda, \lambda, v$ are arbitrary constants. Forming the quotients $A: \Omega, B: \Omega$, and introducing the notations cosh, sinh, of the hyperbolic sine and cosine, also writing for simplicity $v=0$, the equations give

$$
\begin{aligned}
& \sqrt{a-x}=\sqrt{a-b} \cosh \frac{1}{2} u, \\
& \sqrt{b-x}=-\sqrt{a-b} \sinh \frac{1}{2} u,
\end{aligned}
$$

which express the integral of the differential relation

$$
\partial u=\frac{\partial x}{\sqrt{a-x \cdot b-x}}
$$

C. $\mathrm{X}$. 
Instead of considering in like manner the radical $\sqrt{a-x \cdot b-x \cdot c-x}, \mathrm{I}$ pass at once to the radical $\sqrt{a-x \cdot b-x \cdot c-x \cdot d-x}$; and starting from the differential relation

$$
\partial u=\frac{\partial x}{\sqrt{a-x \cdot b-x \cdot c-x \cdot d-x}},
$$

and using the single letters $A, B, C, D, \Omega$ to denote functions of $u, \mathrm{I}$ assume as definitions

$$
\begin{aligned}
& A=\Omega \sqrt{a-x} \\
& B=\Omega \sqrt{b-x} \\
& C=\Omega \sqrt{c-x} \\
& D=\Omega \sqrt{d-x}
\end{aligned}
$$

and another equation to be presently mentioned; $A, B, C, D$ are called 9 -functions, and $\Omega$ is called the $\omega$-function.

But before proceeding further I introduce some locutions which will be useful. In reference to a given set of squares or products, I use the expression a sum of squares to denote the sum of all or any of the squares each multiplied by an arbitrary coefficient; and in like manner a sum of products to denote the sum of all or any of the products each multiplied by an arbitrary coefficient: in particular, the set may consist of a single square or product only, and the sum of squares or products will then denote the single term multiplied by an arbitrary coefficient. In the present case, we have the quantities $\sqrt{a-x}, \sqrt{b-x}, \sqrt{c-x}, \sqrt{d-x}$, and the squares are $a-x, b-x$, etc., which belong all to the same set; but the products (meaning thereby products of two quantities) $\sqrt{a-x \cdot b-x}$, etc., are considered as being each of them a set by itself. A sum of squares is thus a linear function $\lambda+\mu x$, and conversely any such function is a sum of squares; a sum of products means a single term $\nu \sqrt{a-x \cdot b-x}$ (or $\nu \sqrt{a-x \cdot c-x}$, etc., as the case may be), and conversely any such function is a sum of products: the coefficients $\lambda, \mu, \nu$ may depend upon or contain $\Omega$, and in differential expressions ( $\partial u$ being therein considered constant) the coefficients $\lambda, \mu, \nu$ may contain the factor $\partial u$ or $(\partial u)^{2}$-and if convenient we may of course express such factor by writing the coefficients in the form $\lambda \partial u$, or $\lambda(\partial u)^{2}$ etc., as the case may be.

We may now explain very simply the form, as well of the algebraical relations, as of the differential relations of the first and second orders respectively, which connect the functions $A, B, C, D$.

The functions $A^{2}, B^{2}, C^{2}, D^{2}$ are each of them a sum of squares, and hence there exists a linear relation between any three of these squares. But the products $A B$, $A C$, etc., are each of them a sum of products (meaning thereby a single term, as already explained); and hence there is not any linear relation between these products.

Considering the first derived functions $\partial A, \partial B$, etc., these each contain a term in $\partial \Omega$, which however disappears (as is obvious) from the combinations $A \partial B-B \partial A$, 
etc.; and, without in any wise fixing the value of $\Omega$, we in fact find that each of these expressions is a sum of products; the form is, as will appear,

$$
A \partial B-B \partial A=\alpha \Omega^{2} \sqrt{c-x . d-x}=\nu C D, \text { etc.* }
$$

Passing to the second derived functions and forming the combinations $A \partial^{2} A-(\partial A)^{2}$, etc., each of these will contain a multiple of $\Omega \partial^{2} \Omega-(\partial \Omega)^{2}$, but if we assume this expression $\Omega \partial^{2} \Omega-(\partial \Omega)^{2}=\Omega^{2} M$, where $M$ is $=(\partial u)^{2}$ multiplied by a properly determined function of $x$, then it is found that each of the expressions in question $A \partial^{2} A-(\partial A)^{2}$, etc., becomes equal to a sum of squares, that is, to a linear function $\Omega^{2}(\lambda+\mu x)$ : viz. it is equal to a sum of squares formed with the squares $A^{2}, B^{2}, C^{2}, D^{2}$.

The foregoing equation

$$
\Omega \partial^{2} \Omega-(\partial \Omega)^{2}=\Omega^{2} M,
$$

where $M$ has its proper value, is the other equation above referred to, which, with the equations $A=\Omega \sqrt{a-x}$, etc., serves for the definition of the functions $A, B, C, D, \Omega$; it may be mentioned at once that the proper value is

$$
M=\frac{1}{4}(\partial u)^{2}\left\{-2 x^{2}+x(a+b+c+d)+\kappa\right\},
$$

where $\kappa$ is a constant, symmetrical as regards $a, b, c, d$, which may be taken $=0$, but which is better put

$$
=a^{2}+b^{2}+c^{2}+d^{2}-a b-a c-a d-b c-b d-c d .
$$

For the proof of the formula, I introduce and shall in general employ the abbreviations ( $\mathrm{a}, \mathrm{b}, \mathrm{c}, \mathrm{d})$ to denote the differences $a-x, b-x, c-x, d-x$ : the

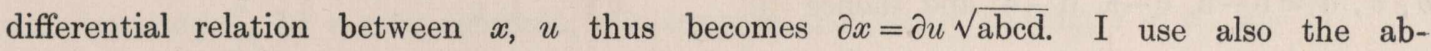
breviations $\Omega \partial^{2} \Omega-(\partial \Omega)^{2}=\Delta \Omega$, etc.

We have

$$
A \partial B-B \partial A=\Omega^{2}(\sqrt{\bar{a}} \partial \sqrt{\bar{b}}-\sqrt{\mathrm{b}} \partial \sqrt{\bar{a}}),
$$

the terms in $\partial \Omega$ disappearing: viz. observing that $\partial \mathrm{a}=\partial \mathrm{b}=-\partial x$, this is

$$
\begin{aligned}
& =-\frac{1}{2} \Omega^{2}\left(\frac{\sqrt{ } \mathrm{a}}{\sqrt{b}}-\frac{\sqrt{ } b}{\sqrt{ } a}\right) \partial x, \\
& =\quad \frac{1}{2} \Omega^{2} \frac{a-b}{\sqrt{a b}} \partial x
\end{aligned}
$$

or observing that $a-b=a-b$, and writing for $\partial x$ its value $=\sqrt{a b c d} \partial u$, this is

$$
\begin{aligned}
A \partial B-B \partial A & =-\frac{1}{2}(a-b) \Omega^{2} \sqrt{c d} \partial u, \\
& =-\frac{1}{2}(a-b) \Omega^{2} \sqrt{c-x . a-x} \partial u,
\end{aligned}
$$

which is the equation expressing $A \partial B-B \partial A$ as a sum of products: it is further obvious that the value is

$$
=-\frac{1}{2}(a-b) C D \partial u \text {. }
$$
cases.

* It is hardly necessary to remark that $a, \nu$ contain each of them the factor $\partial u$; and the like in other 
Proceeding next to find the value of $\Delta A,=A \partial^{2} A-(\partial A)^{2},=A^{2} \partial^{2} \log A$, it is to be remarked that we have in general

$$
\Delta P Q=P^{2} \Delta Q+Q^{2} \Delta P,
$$

and therefore also $\Delta P^{2}=2 P^{2} \Delta P$, and consequently $\Delta \sqrt{P}=\frac{\frac{1}{2}}{P} \Delta P$. Hence starting from $A=\Omega \sqrt{ }$ a, we have

$$
\Delta A=\mathrm{a} \Delta \Omega+\Omega^{2} \frac{\frac{1}{2}}{\mathrm{a}} \Delta \mathrm{a}
$$

where $\Delta \mathrm{a}=-\mathrm{a} \partial^{2} x-(\partial x)^{2}$. I assume that we have $\Delta \Omega=\Omega^{2} M=\frac{1}{4} \Omega^{2} S(\partial u)^{2}$, where $S$ denotes a function of $x$ which is to be determined: the equation thus becomes

$$
\Delta A=\frac{1}{4} \Omega^{2}\left\{a S(\partial u)^{2}-2 \partial^{2} x-2(\partial x)^{2}\right\} ;
$$

we have $(\partial x)^{2}=\operatorname{abcd}(\partial u)^{2}$, and thence, differentiating and omitting on each side the factor $\partial x$, we obtain

$$
2 \partial^{2} x=-(a b c+a b d+a c d+b c d)
$$

and the equation becomes

$$
\Delta A=\frac{1}{4} \Omega^{2}\{\mathrm{a}(S+\mathrm{bc}+\mathrm{bd}+\mathrm{cd})-\mathrm{bcd}\}(\partial u)^{2},
$$

which is to be simplified by assuming a proper value for $S$; in order that the same simplification may apply to the formulæ for $\Delta B$, etc., it is necessary that $S$ be symmetrical in regard to $a, b, c, d$.

Writing for the moment $b^{\prime}, c^{\prime}, d^{\prime}$ to denote $b-a, c-a, d-a$ respectively, we have $b^{\prime}, c^{\prime}, d^{\prime}=\mathrm{b}-\mathrm{a}, \mathrm{c}-\mathrm{a}, \mathrm{d}-\mathrm{a}$, and thence

and consequently

$$
b^{\prime} c^{\prime} d^{\prime}=b c d-a(b c+b d+c d)+a^{2}(b+c+d)-a^{3},
$$

$$
a(b c+b d+c d)-b c d=-b^{\prime} c^{\prime} d^{\prime}+a^{2}(b+c+d-a):
$$

hence, in the expression of $\Delta A$, the factor which multiplies $\frac{1}{2} \Omega^{2}(\partial u)^{2}$ is

$$
a\{S+a(b+c+d-a)\}-b^{\prime} c^{\prime} d^{\prime},
$$

viz. the expression added to $S$ is

$$
\begin{gathered}
(a-x)(b+c+d-a-2 x), \\
=a(b+c+d-a)-x(a+b+c+d)+2 x^{2} \\
S=-2 x^{2}+x(a+b+c+d)+\kappa
\end{gathered}
$$

$\kappa$ being a constant symmetrical in regard to $a, b, c, d$, which may be at once taken to be $=a^{2}+b^{2}+c^{2}+d^{2}-a b-a c-a d-b c-b d-c d$; then writing also

$$
\lambda=b^{2}+c^{2}+d^{2}-b c-b d-c d, \quad \mu=-b^{\prime} c^{\prime} d^{\prime}=a-b . a-c . a-d,
$$

the expression a $\{S+\mathrm{a}(\mathrm{b}+\mathrm{c}+\mathrm{d}-\mathrm{a})\}-b^{\prime} c^{\prime} d^{\prime}$ becomes $=\mathrm{a} \lambda+\mu$; and the sought for equation thus is

$$
\Delta A=A \partial^{2} A-(\partial A)^{2}=\frac{1}{4} \Omega^{2}(\mathrm{a} \lambda+\mu)(\partial u)^{2},
$$


the equation in $\Omega$ being of course

$$
\Delta \Omega=\Omega \partial^{2} \Omega-(\partial \Omega)^{2}=\frac{1}{4} \Omega^{2}\left\{-2 x^{2}+x(a+b+c+d)+\kappa\right\}(\partial u)^{2} .
$$

The theory in regard to the second derivatives is thus completed.

To adapt the formulæ to elliptic integrals, and ordinary $H$ and $\Theta$ functions, the radical must be brought to the form $\sqrt{x .1-x .1-k^{2} x}$. Writing for this purpose

$$
a, b, c, d=-k^{2} I^{2}, 0,1, \frac{1}{k^{2}},(I=\infty),
$$

substituting also $\frac{2 u}{I}$ for $u$, and $i k I . A, i B(i=\sqrt{-1}$ as usual) for $A, B$ respectively, we find $\sqrt{a-x . b-x . c-x . d-x}=I \sqrt{x .1-x .1-k^{2} x}$; and then

$$
2 \partial u=\frac{\partial x}{\sqrt{x .1-x .1-k^{2} x}}
$$

and

$$
A=\Omega, B=\Omega \sqrt{x}, C=\Omega \sqrt{1-x}, D=\frac{1}{k} \Omega \sqrt{1-k^{2} x}
$$

$\Omega$ is in this case $=A$, a 9 -function : and in the equation for $\Delta \Omega$, writing $A$ in place of $\Omega$, the equation becomes

$$
A \partial^{2} A-(\partial A)^{2}=\frac{1}{4} A^{2}\left\{-2 x^{2}+x\left(-k^{2} I^{2}\right)+\kappa\right\} \frac{4(\partial u)^{2}}{I^{2}}
$$

viz. replacing $\frac{\kappa}{I^{2}}$ by a new constant, $=\lambda$ suppose and finally putting $I=\infty$, this is

$$
A \partial^{2} A-(\partial A)^{2}=A^{2}\left(\lambda-k^{2} x\right)(\partial u)^{2} .
$$

The differential equation is satisfied by $x=\mathrm{sn}^{2} u$, giving $1-x=\mathrm{cn}^{2} u, 1-k^{2} x=\mathrm{dn}^{2} u$; and the equation for $A$ then is

or say

$$
\partial^{2} \log A=\left(\lambda-k^{2} \operatorname{sn}^{2} u\right)(\partial u)^{2},
$$

$$
A=L e^{\frac{1}{2} \lambda u^{2}-k^{2} \int_{0} d u \int_{0} d u \operatorname{sn}^{2} u}
$$

viz. by properly assuming the constants $L, \lambda$, we shall have $A=$ Jacobi's function $\Theta u$ : and then sn $u=\frac{B}{A}$, cn $u=\frac{C}{A}, \operatorname{dn} u=\frac{k D}{A}$, which will give the ordinary expressions of sn, cn, dn in terms of $H, \Theta$.

\section{Part II. The double 9-functions.}

Passing now to the double 9 -functions, and writing for a moment

$$
\begin{aligned}
& \sqrt{X}=\sqrt{a-x \cdot b-x \cdot c-x \cdot d-x \cdot e-x \cdot f-x} \\
& \sqrt{Y}=\sqrt{a-y \cdot b-y \cdot c-y \cdot d-y \cdot e-y \cdot f-y}
\end{aligned}
$$


the differential equations which connect $u, v$ with $x, y$ are

$$
\begin{aligned}
& \partial u=\frac{\partial x}{\sqrt{ } X}+\frac{\partial y}{\sqrt{ } Y} \\
& \partial v=\frac{x \partial x}{\sqrt{ } X}+\frac{y \partial y}{\sqrt{ } Y}
\end{aligned}
$$

There are here sixteen 9 -functions $A, B, C, D, E, F, A B, A C, A D, A E, B C, B D, B E$, $C D, C E, D E$, and an associated $\omega$-function $\Omega$, where for shortness $\mathrm{I}$ use the single and double letters $A, B, \ldots, A B, \ldots, \Omega$, instead of functional expressions $A(u, v), B(u, v), \ldots$, $A B(u, v), ., \Omega(u, v)$, to denote functions of the two letters $u, v$. Writing also ( $\mathrm{a}, \mathrm{b}, \mathrm{c}, \mathrm{d}, \mathrm{e}, \mathrm{f})$ for the differences $a-x, b-x$, etc., and $\left(\mathrm{a}_{1}, \mathrm{~b}_{1}, \mathrm{c}_{1}, \mathrm{~d}_{1}, \mathrm{e}_{1}, \mathrm{f}_{1}\right)$ for the

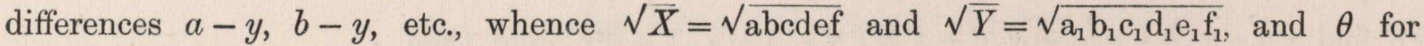
the difference $x-y$, we have sixteen $x y$-functions which are represented by

$$
\sqrt{ } a, \sqrt{ } b, \sqrt{ } c, \sqrt{ } d, \sqrt{ } e, \sqrt{ } f, \sqrt{a b}, \sqrt{a c}, \sqrt{a d}, \sqrt{a e}, \sqrt{b c}, \sqrt{b d}, \sqrt{b e}, \sqrt{c d}, \sqrt{c e}, \sqrt{d e},
$$

the values of which are

$$
\begin{aligned}
\sqrt{ } a & =\sqrt{a a_{1}}, \text { (six equations), } \\
\vdots & \\
\sqrt{a b} & =\frac{1}{\theta}\left\{\sqrt{a b f_{1} d_{1} e_{1}}-\sqrt{a_{1} b_{1} f_{1} c d e}\right\}, \text { (ten equations), }
\end{aligned}
$$

and the definitions of the sixteen 9 -functions and the $\omega$-function are

$$
\begin{gathered}
A=\Omega \sqrt{ } a, \quad \text { (six equations), } \\
\vdots \\
A B=\Omega \sqrt{a b}, \text { (ten equations), }
\end{gathered}
$$

and one other equation to be afterwards mentioned.

I call to mind that, in a binary symbol such as $\sqrt{a b}$, it is always $f$ that accompanies the two expressed letters $a, b$ : the duad $a b$ is, in fact, an abbreviated expression for the double triad $a b f . c d e$ : and I remark also that I have for greater simplicity omitted certain constant factors which, in my second paper above referred to, were introduced as multipliers of the foregoing functions $\sqrt{ } a, \ldots, \sqrt{a b}, \ldots$ I remark also that, to avoid confusion, the square of any one of these functions $\sqrt{a}$ or $\sqrt{a b}$ is always written (not $a$ or $a b$, but) $(\sqrt{ } a)^{2}$ or $(\sqrt{a b})^{2}$.

I use $\partial$ as a symbol of total differentiation: thus

$$
\partial A=\frac{d A}{d u} \partial u+\frac{d A}{d v} \partial v, \quad \partial^{2} A=\frac{d^{2} A}{d u^{2}}(\partial u)^{2}+2 \frac{d^{2} A}{d u d v}(\partial u \partial v)+\frac{d^{2} A}{d v^{2}}(\partial v)^{2}, \text { etc. }
$$

Moreover I consider $\partial u$ and $\partial v$ as constants, and use single letters $\lambda$, $L$, etc., to denote linear functions $\alpha \partial u+\beta \partial v$, or quadric functions $\alpha(\partial u)^{2}+2 \beta \partial u \partial v+\gamma(\partial v)^{2}$ (as the 
case may be) of these differentials; thus, in speaking of $A \partial B-B \partial A$ as a sum of products, it is implied that the coefficients of the several products are linear functions of $\partial u, \partial v$; and so in speaking of $A \partial^{2} A-(\partial A)^{2}$ as a sum of squares, it is in like manner implied that the coefficients of the several squares are quadric functions of $\partial u, \partial v$.

An $x y$-function is simplex, such as $\sqrt{ } a$, or complex, such as $\sqrt{a b}$; the square of the former is $\mathrm{aa}_{1}=a^{2}-a(x+y)+x y$, which is of the form $\lambda+\mu(x+y)+\nu x y$; the square of the latter is

$$
=\frac{1}{\theta^{2}}\left\{a b f c_{1} d_{1} e_{1}+a_{1} b_{1} f_{1} c d e-2 \sqrt{X Y}\right\}
$$

where observe that the irrational part $-\frac{2}{\theta^{2}} \sqrt{X Y}$ is the same for all these squares: so that, taking any two such squares, their difference is $=\frac{1}{\theta^{2}}$ multiplied by a rational function of $x y$ : this rational function in fact divides by $\theta^{2}$, the quotient being a rational and integral function of the foregoing form $\lambda+\mu(x+y)+\nu x y$. Hence selecting any one of the complex functions, say $\sqrt{d e}$, the square of any other of the complex functions is equal to the square of this plus a term $\lambda+\mu(x+y)+\nu x y$; and hence the square of any function simplex or complex is of the form $\lambda+\mu(x+y)+\nu x y+\rho(\sqrt{d e})^{2}$; this being so, the squares of the $x y$-functions may be regarded as forming a single set; every sum of squares is a function of this form $\lambda+\mu(x+y)+\nu x y+\rho(\sqrt{d e})^{2}$; and conversely every function of this form is a sum of squares. A sum of squares thus depends upon four arbitrary coefficients $\lambda, \mu, \nu, \rho$; and we may, in an infinity of ways, select four out of the 16 squares such that every sum of squares can be represented as a sum of these four squares each multiplied by the proper coefficient; say as a sum of the selected four squares: in particular, each of the remaining squares can be expressed as a sum of the selected four squares. It appears, by the first of my papers above referred to, that there are systems of four squares connected together by a linear equation: we are not here concerned with such systems; only of course the four selected squares must not belong to such a system.

We have the products of the $x y$-functions, where by product is meant a product of two functions. The number of products is of course $=120$, but distinguishing these according to the radicals which they respectively contain, they form 30 different sets. Thus we have

$$
\begin{aligned}
& \sqrt{ } b \sqrt{a b}=\frac{1}{\theta}\left\{b \sqrt{a f b_{1} c_{1} d_{1} e_{1}}-b_{1} \sqrt{a_{1} f_{1} b c d e}\right\} \\
& \sqrt{c} \sqrt{a c}=\frac{1}{\theta}\left\{c \quad \# \quad-c_{1} \quad \# \quad\right\}, \\
& \sqrt{ } d \sqrt{a d}=\frac{1}{\theta}\left\{\mathrm{d} \quad, \quad-\mathrm{d}_{1} \quad, \quad\right\}, \\
& \sqrt{e} \sqrt{a e}=\frac{1}{\theta}\left\{\mathrm{e} \quad \#-\mathrm{e}_{1} \quad, \quad\right\},
\end{aligned}
$$


which four expressions form a set, and there are 15 such sets. The set written down may be called the set af: and the fifteen sets are of course ab, ac, etc.

Again, we have

$$
\begin{aligned}
& \sqrt{ } a \sqrt{ } b=\quad \sqrt{a b a_{1} b_{1}}, \\
& \sqrt{a c} \sqrt{b c}=\frac{1}{\theta^{2}}\left\{\left(\operatorname{cfd}_{1} \mathrm{e}_{1}+c_{1} \mathrm{f}_{1} d e\right) \sqrt{\mathrm{aba}_{1} \mathrm{~b}_{1}}-\left(a b_{1}+\mathrm{a}_{1} \mathrm{~b}\right) \sqrt{\operatorname{cdefc}_{1} \mathrm{~d}_{1} \mathrm{e}_{1} \mathrm{f}_{1}}\right\}, \\
& \sqrt{a d} \sqrt{b d}=\frac{1}{\theta^{2}}\left\{\left({d f c_{1}}_{1} e_{1}+d_{1} f_{1} c e\right) \quad, \quad \text { " }\right\}, \\
& \sqrt{a e} \sqrt{b e}=\frac{1}{\theta^{2}}\left\{\left(\operatorname{efc}_{1} \mathrm{~d}_{1}+\mathrm{e}_{1} \mathrm{f}_{1} \mathrm{~cd}\right) \quad, \quad \text { " } \quad,\right\},
\end{aligned}
$$

which four expressions form a set, and there are 15 such sets. The set written down may be called the set $a b a_{1} b_{1}$ : and the fifteen sets are of course $a b a_{1} b_{1}$, aca $c_{1}$, etc. The 15 and 15 sets make in all 30 sets as mentioned above.

The expression, a sum of products, means as already explained a sum of products belonging to the same set; and there are thus 30 forms of a sum of products. The products of the same set are connected by two linear relations, so that, selecting at pleasure any two of the products, the other two products can be expressed each of them as a linear function of these; hence a sum of products contains only two arbitrary coefficients.

Reverting now to the equations $A=\Omega \sqrt{ } a$, etc., we see at once the form of the algebraical equations which connect the 16 -functions. Every squared function $A^{2}, \ldots,(A B)^{2}, \ldots$ is a sum of squares, whence selecting (as may be done in a great number of ways) four of these squared functions, each of the remaining 12 squares is a sum of these four squares each multiplied by the proper coefficient; or say it is a sum of the four selected squares. And in like manner the 120 products of two of the 16 functions form 30 sets, such that selecting at pleasure two of the set, the remaining two of the set are each of them a linear function of these.

Considering the first derived functions $\partial A, \partial B, \ldots, \partial A B, \ldots$, each of these contains a term in $\partial \Omega$; but $\partial \Omega$ disappears (as is obvious) from the several combinations $I \partial J-J \partial I$ (I write $I$ and similarly $J$ to denote indifferently a single letter $A$ or a double letter $A B)$ : and, without in any wise fixing the value of $\Omega$, we in fact find that each of these expressions is a sum of products.

Passing to the second derived functions, and forming the combinations $A \partial^{2} A-(\partial A)^{2}$, etc., or to include the two cases of the single and the double letter, say $I \partial^{2} I-(\partial I)^{2}$, each of these will contain a multiple of $\Omega \partial^{2} \Omega-(\partial \Omega)^{2}$; but if we assume this expression $\Omega \partial^{2} \Omega-(\partial \Omega)^{2}=\Omega^{2} M$, where $M$ is a quadric function of $\partial u, \partial v$, the coefficients of $(\partial u)^{2}, \partial u \partial v$, $(\partial v)^{2}$ being properly determined functions of $x y$, then it is found that each of the expressions in question $I \partial^{2} I-(\partial I)^{2}$ becomes equal to a sum of squares.

It is to be observed that $M$ is not altogether arbitrary: the equation as containing terms in $(\partial u)^{2}, \partial u \partial v$, and $(\partial v)^{2}$, in fact represents three partial differential 
equations, which for an arbitrary value of $M$ would be inconsistent with each other: it is therefore necessary to verify that the value assigned to $M$ is such as to render the three equations consistent with each other, and this will accordingly be done.

The foregoing equation

$$
\Omega \partial^{2} \Omega-(\partial \Omega)^{2}=\Omega^{2} M,
$$

where $M$ has its proper value, (or say the three partial differential equations into which this breaks up), constitutes the other equation above referred to, which with the original equations $A=\Omega \sqrt{ } a$, etc., serve to define the sixteen 9 -functions and $\Omega$.

The remainder of the present memoir is occupied with the analytical investigation of the foregoing theorems. Although the mere algebraical work is very long, yet it appears to me interesting, and I have thought it best to give it in detail.

The analytical theory: various subheadings.

The equations

$$
\begin{aligned}
& \partial u=\frac{\partial x}{\sqrt{ } X}+\frac{\partial y}{\sqrt{ } Y} \\
& \partial v=\frac{x \partial x}{\sqrt{ } X}+\frac{y \partial y}{\sqrt{ } Y}
\end{aligned}
$$

give

$$
\frac{\theta \partial x}{\sqrt{X}}=\partial v-y \partial u, \quad-\frac{\theta \partial y}{\sqrt{Y}}=\partial v-x \partial u
$$

which determine $\partial x, \partial y$ in terms of $\partial u, \partial v$. A different form is sometimes convenient; writing $\partial \varpi=\partial v-a \partial u$, and recollecting that a, $a_{1}$ denote $a-x, a-y$ respectively, the equations become

$$
\frac{\theta \partial x}{\sqrt{ } X}=\partial \varpi+\mathrm{a}_{1} \partial u, \quad-\frac{\theta \partial y}{\sqrt{ } Y}=\partial \varpi+\mathrm{a} \partial u
$$

\section{Expression for $\partial \sqrt{ } a$.}

We have

$$
\begin{aligned}
\partial \sqrt{ } a & =\partial \sqrt{\mathrm{aa}_{1}}=\frac{1}{2 \sqrt{\mathrm{aa}_{1}}}\left(\mathrm{a} \partial \mathrm{a}_{1}+\mathrm{a}_{1} \partial \mathrm{a}\right)=-\frac{1}{2 \sqrt{\mathrm{aa}_{1}}}\left(\mathrm{a} \partial y+\mathrm{a}_{1} \partial x\right) \\
& =\frac{1}{2 \sqrt{\mathrm{aa}_{1}}} \frac{1}{\theta}\left\{\mathrm{a} \sqrt{Y}(\partial v-x \partial u)-\mathrm{a}_{1} \sqrt{X}(\partial v-y \partial u)\right\}
\end{aligned}
$$

substituting for $\sqrt{ } X, \sqrt{ } Y$ their values $\sqrt{\text { abcdef, }} \sqrt{\mathrm{a}_{1} \mathrm{~b}_{1} \mathrm{c}_{1} \mathrm{~d}_{1} \mathrm{e}_{1} \mathrm{f}_{1}}$, this is

$$
\partial \sqrt{ } a=\frac{\frac{1}{2}}{\theta}\left\{\sqrt{\mathrm{ab}_{1} \mathrm{c}_{1} \mathrm{~d}_{1} \mathrm{e}_{1} \mathrm{f}_{1}}(\partial v-x \partial u)-\sqrt{\mathrm{a}_{1} \mathrm{bcdef}}(\partial v-y \partial u)\right\}
$$

and by the mere interchange of letters we can of course find $\partial \sqrt{ } b$, etc.

c. $\mathrm{x}$. 
Expression for $\partial \sqrt{a b}$.

We have next to find

$$
\partial \sqrt{a b}=\partial \frac{1}{\theta}\left\{\sqrt{a b f c_{1} d_{1} e_{1}}-\sqrt{a_{1} b_{1} f_{1} c d e}\right\} ;
$$

here

$$
\partial \theta=\partial x-\partial y, \quad=\frac{1}{\theta}\left\{\sqrt{\operatorname{abcdef}}(\partial v-y \partial u)+\sqrt{\mathrm{a}_{1} \mathrm{~b}_{1} \mathrm{c}_{1} \mathrm{~d}_{1} \mathrm{e}_{1} \mathrm{f}_{1}}(\partial v-x \partial u)\right\}
$$

and consequently $\partial \sqrt{a b}$ contains a term

which is

$$
-\frac{\partial \theta}{\theta^{2}}\left\{\sqrt{a b f c_{1} d_{1} e_{1}}-\sqrt{a_{1} b_{1} f_{1} c d e}\right\}
$$

$$
\begin{gathered}
=\frac{1}{\theta^{3}}\left\{\left(-a b f \sqrt{\operatorname{cdec}_{1} d_{1} e_{1}}+\text { cde } \sqrt{a b f a_{1} b_{1} f_{1}}\right)(\partial v-y \partial u)\right. \\
\left.+\left(-c_{1} d_{1} e_{1} \sqrt{a b f a_{1} b_{1} f_{1}}+a_{1} b_{1} f_{1} \sqrt{\operatorname{coc}_{1} d_{1} e_{1}}\right)(\partial v-x \partial u)\right\},
\end{gathered}
$$

or, what is the same thing,

Now

$$
\begin{aligned}
& =\frac{1}{\theta^{2}}\left\{\sqrt{\operatorname{abfa}_{1} b_{1} f_{1}}\left(\frac{\text { cde }-c_{1} d_{1} e_{1}}{\theta} \partial v+\frac{-\operatorname{cde} y+c_{1} d_{1} e_{1} x}{\theta} \partial u\right)\right. \\
& \left.+\sqrt{\operatorname{cdec}_{1} \mathrm{~d}_{1} \mathrm{e}_{1}}\left(\frac{-\mathrm{abf}+\mathrm{a}_{1} \mathrm{~b}_{1} \mathrm{f}_{1}}{\theta} \partial v+\frac{\mathrm{abf} y-\mathrm{a}_{1} \mathrm{~b}_{1} \mathrm{f}_{1} x}{\theta} \partial u\right)\right\} .
\end{aligned}
$$

$$
\begin{aligned}
\frac{c d e-c_{1} d_{1} e_{1}}{\theta} & =-(c d+c e+d e)+(c+d+e)(x+y)-x^{2}-x y-y^{2} \\
\frac{-\operatorname{cde} y+c_{1} d_{1} e_{1} x}{\theta} & =c d e-(c+d+e) x y+x y(x+y)
\end{aligned}
$$

with the like formulæ with $\mathrm{a}, \mathrm{b}, \mathrm{f}$ instead of $\mathrm{c}, \mathrm{d}$, e. Hence the foregoing, or say the first, part of $\partial \sqrt{a b}$ is

$$
\begin{gathered}
=\frac{1}{\theta^{2}}\left[\sqrt { \mathrm { abfa } _ { 1 } \mathrm { b } _ { 1 } \mathrm { f } _ { 1 } } \left(\left\{-(c d+c e+d e)+(c+d+e)(x+y)-x^{2}-x y-y^{2}\right\} \partial v\right.\right. \\
+\{c d e-(c+d+e) x y+x y(x+y)\} \partial u) \\
+\sqrt{\operatorname{cdec}_{1} \mathrm{~d}_{1} \mathrm{e}_{1}}\left(\left\{a b+a f+b f-(a+b+f)(x+y)+x^{2}+x y+y^{2}\right\} \partial v\right. \\
+\{-a b f+(a+b+f) x y-x y(x+y)\} \partial u)] .
\end{gathered}
$$

The other or second part of $\partial \sqrt{a b}$, using for shortness an accent to denote differentiation in regard to $x$ or to $y$, according as it is applied to a function of $x$ or of $y$, is readily found to be

$$
\begin{aligned}
= & \frac{\frac{1}{2}}{\theta^{2}}\left[\sqrt{a b a_{1} b_{1} \mathrm{f}_{1}}\left(\left\{-(\text { cde })^{\prime}-\left(c_{1} \mathrm{~d}_{1} \mathrm{e}_{1}\right)^{\prime}\right\} \partial v+\left\{y(\text { cde })^{\prime}+x\left(c_{1} \mathrm{~d}_{1} \mathrm{e}_{1}\right)^{\prime}\right\} \partial u\right)\right. \\
& \left.+\sqrt{\operatorname{cdec}_{1} \mathrm{~d}_{1} \mathrm{e}_{1}}\left(\left\{(\mathrm{abf})^{\prime}+\left(\mathrm{a}_{1} \mathrm{~b}_{1} \mathrm{f}_{1}\right)^{\prime}\right\} \partial v+\left\{-y(\mathrm{abf})^{\prime}-x\left(\mathrm{a}_{1} \mathrm{~b}_{1} \mathrm{f}_{1}\right)^{\prime}\right\} \partial u\right)\right] .
\end{aligned}
$$


Hence uniting the two terms so as to form the complete value of $\partial \sqrt{a b}$, we have first, a term $\frac{1}{\theta^{2}} \sqrt{\mathrm{abfa}_{1} \mathrm{~b}_{1} \mathrm{f}_{1}} \partial v$, the coefficient of which is

$$
\begin{aligned}
= & -(c d+c e+d e)+(c+d+e)(x+y)-x^{2}-x y-y^{2} \\
& -\frac{1}{2}\left\{(c d e)^{\prime}+\left(c_{1} d_{1} \mathrm{e}_{1}\right)^{\prime}\right\}:
\end{aligned}
$$

this second line is

$$
=c d+c e+d e-(c+d+e)(x+y)+\frac{3}{2} x^{2}+\frac{3}{2} y^{2},
$$

or the coefficient is $-\frac{1}{2} x^{2}+x y-\frac{1}{2} y^{2},=-\frac{1}{2} \theta^{2}$; the term is thus

$$
=\frac{1}{2} \sqrt{\mathrm{abfa}_{1} \mathrm{~b}_{1} \mathrm{f}_{1}} \partial v \text {. }
$$

Secondly, a term in $\frac{1}{\theta^{2}} \sqrt{\operatorname{cdec}_{1} \mathrm{~d}_{1} \mathrm{e}_{1}} \partial v$ which is in like manner found to be

$$
=-\frac{1}{2} \sqrt{\operatorname{cdec}_{1} \mathrm{~d}_{1} \mathrm{e}_{1}} \partial v \text {. }
$$

Thirdly, a term $\frac{1}{\theta^{2}} \sqrt{\mathrm{abfa}_{1} \mathrm{~b}_{1} \mathrm{f}_{1}} \partial u$, the coefficient of which is

this second line is

$$
\begin{gathered}
=c d e-(c+d+e) x y+x^{2} y+x y^{2} \\
+\frac{1}{2}\left\{y(c d e)^{\prime}+x\left(c_{1} \mathrm{~d}_{1} \mathrm{e}_{1}\right)^{\prime}\right\}:
\end{gathered}
$$

$$
=-(c d+c e+d e) \frac{1}{2}(x+y)+(c+d+e) 2 x y-\frac{3}{2} x^{2} y-\frac{3}{2} x y^{2},
$$

and the coefficient is thus

$$
\begin{aligned}
=\frac{1}{2}\{2 c d e-(c d+c e+d e)(x+y) & +(c+d+e)\left(x^{2}+y^{2}\right)-x^{3} \\
- & -y^{3} \\
& \left.(c+d+e)(x-y)^{2}+x^{3}-x^{2} y-x y^{2}+y^{3}\right\},
\end{aligned}
$$

which is

or the term is

$$
=\frac{1}{2}\left\{c d e+c_{1} d_{1} \mathrm{e}_{1}-(c+d+e) \theta^{2}+(x+y) \theta^{2}\right\},
$$

$$
=\frac{1}{2} \sqrt{\mathrm{abfa}_{1} \mathrm{~b}_{1} \mathrm{f}_{1}}\left\{\frac{c d e+\mathrm{c}_{1} \mathrm{~d}_{1} \mathrm{e}_{1}}{\theta^{2}}-(c+d+e)+x+y\right\} \partial u \text {. }
$$

And, fourthly, a term in $\frac{1}{\theta^{2}} \sqrt{\operatorname{coc}_{1} \mathrm{~d}_{1} \mathrm{e}_{1}} \partial u$, which is in like manner found to be

$$
=-\frac{1}{2} \sqrt{\operatorname{cdec}_{1} \mathrm{~d}_{1} \mathrm{e}_{1}}\left\{\frac{\mathrm{abf}+\mathrm{a}_{1} \mathrm{~b}_{1} \mathrm{f}_{1}}{\theta^{2}}-(a+b+f)+x+y\right\} \partial u \text {. }
$$

Hence combining these several terms, we have finally

$$
\begin{aligned}
\partial \sqrt{a b} & =\frac{1}{2} \sqrt{a \operatorname{afa}_{1} \mathrm{~b}_{1} \mathrm{f}_{1}}\left[\partial v+\left(\frac{c d e+c_{1} \mathrm{~d}_{1} \mathrm{e}_{1}}{\theta^{2}}-c-d-e+x+y\right) \partial u\right] \\
& +\frac{1}{2} \sqrt{\operatorname{cdec}_{1} \mathrm{~d}_{1} \mathrm{e}_{1}}\left[-\partial v-\left(\frac{a b f+\mathrm{a}_{1} \mathrm{~b}_{1} \mathrm{f}_{1}}{\theta^{2}}-a-b-f+x+y\right) \partial u\right]
\end{aligned}
$$

and by the mere interchange of letters we can of course find $\partial \sqrt{a c}$, etc. 


\section{Expression for $A \partial B-B \partial A$.}

Starting now from the equations $A=\Omega \sqrt{ } a, B=\Omega \sqrt{ } b$, we obtain $A \partial B-B \partial A=\Omega^{2}\{\sqrt{ } a \partial \sqrt{ } b-\sqrt{ } b \partial \sqrt{ } a\}$

$$
\begin{aligned}
= & \frac{\frac{1}{2} \Omega^{2}}{\theta}\left(\sqrt{\mathrm{aa}_{1}}\left\{\sqrt{\mathrm{ba}_{1} \mathrm{c}_{1} \mathrm{~d}_{1} \mathrm{e}_{1} \mathrm{f}_{1}}(\partial v-x \partial u)-\sqrt{\mathrm{b}_{1} \mathrm{acdef}}(\partial v-y \partial u)\right\}\right. \\
& \left.-\sqrt{\mathrm{bb}_{1}}\left\{\sqrt{\mathrm{ab}_{1} \mathrm{c}_{1} \mathrm{~d}_{1} \mathrm{e}_{1} \mathrm{f}_{1}}(\quad, \quad)-\sqrt{\mathrm{a}_{1} \mathrm{bcdef}}(\quad, \quad)\right\}\right), \\
= & \frac{\frac{1}{2} \Omega^{2}}{\theta}\left\{\left(\mathrm{a}_{1}-\mathrm{b}_{1}\right) \sqrt{\mathrm{abc}_{1} \mathrm{~d}_{1} \mathrm{e}_{1} \mathrm{f}_{1}}(\partial v-x \partial u)-(\mathrm{a}-\mathrm{b}) \sqrt{\mathrm{a}_{1} \mathrm{~b}_{1} \operatorname{cdef}}(\partial v-y \partial u)\right\} ;
\end{aligned}
$$

or since $a_{1}-b_{1}=a-b=a-b$, this is

$$
A \partial B-B \partial A=\frac{\frac{1}{2} \Omega^{2}}{\theta}(a-b)\left\{\sqrt{\operatorname{abc}_{1} \mathrm{~d}_{1} \mathrm{e}_{1} \mathrm{f}_{1}}(\partial v-x \partial u)-\sqrt{\mathrm{a}_{1} \mathrm{~b}_{1} \operatorname{cdef}}(\partial v-y \partial u)\right\}
$$

which is a sum of products of the set $a b$ : in fact, the four products of this set are

$$
\begin{aligned}
& \sqrt{ } f \sqrt{a b}=\frac{1}{\theta}\left\{f \sqrt{a b c_{1} d_{1} e_{1} f_{1}}-f_{1} \sqrt{a_{1} b_{1} c d e f}\right\}, \\
& \sqrt{ } c \sqrt{d e}=\frac{1}{\theta}\left\{-\mathrm{c} \quad, \quad+\mathrm{c}_{1} \quad \# \quad\right\}, \\
& \sqrt{ } d \sqrt{c e}=\frac{1}{\theta}\left\{-\mathrm{d} \quad, \quad+\mathrm{d}_{1} \quad \# \quad\right\}, \\
& \sqrt{e} \sqrt{c d}=\frac{1}{\theta}\left\{-\mathrm{e} \quad, \quad+\mathrm{e}_{1} \quad \# \quad\right\}
\end{aligned}
$$

choosing any two of these at pleasure, for instance the first and second, multiplying by $\partial v-c \partial u, \partial v-f \partial u$ and adding, we have

$$
\left.\begin{array}{r}
(\partial v-c \partial u) \sqrt{ } f \sqrt{a b} \\
+(\partial v-f \partial u) \sqrt{ } c \sqrt{d e}
\end{array}\right\}=\left\{\begin{array}{l}
\frac{1}{\theta}\left\{(\partial v-c \partial u) f \sqrt{\mathrm{abc}_{1} \mathrm{~d}_{1} \mathrm{e}_{1} \mathrm{f}_{1}}-(\partial v-c \partial u) \mathrm{f}_{1} \sqrt{\left.\mathrm{a}_{1} \mathrm{~b}_{1} \mathrm{cdef}\right\}}\right. \\
\frac{1}{\theta}\left\{-(\partial v-f \partial u) c \quad " \quad+(\partial v-f \partial u) \mathrm{c}_{1} \quad " \quad\right\}
\end{array}\right.
$$

where the coefficients $\mathrm{f}(\partial v-c \partial u)-\mathrm{c}(\partial v-f \partial u)$, and $\mathrm{f}_{1}(\partial v-c \partial u)-\mathrm{c}_{1}(\partial v-f \partial u)$, by substituting for $\mathrm{f}, \mathrm{c}, \mathrm{f}_{1}, \mathrm{c}_{1}$ their values, become $=(f-c)(\partial v-x \partial u)$ and $(f-c)(\partial v-y \partial u)$; and the expression is thus

$$
=\frac{f-c}{\theta}\left\{\sqrt{\mathrm{abc}_{1} \mathrm{~d}_{1} \mathrm{e}_{1} \mathrm{f}_{1}}(\partial v-x \partial u)-\sqrt{\mathrm{a}_{1} \mathrm{~b}_{1} \operatorname{cdef}}(\partial v-y \partial u)\right\} .
$$

Reverting to the original expression for $A \partial B-B \partial A$, it may be remarked that, if we write $\partial v-a \partial u=\partial \varpi, \partial v-b \partial u=\partial \sigma$, then

$$
(a-b)(\partial v-x \partial u)=a \partial \sigma-b \partial \varpi, \quad(a-b)(\partial v-y \partial u)=a_{1} \partial \sigma-b_{1} \partial \varpi
$$


and the formula thus becomes

$$
A \partial B-B \partial A=\frac{\frac{1}{2} \Omega^{2}}{\theta}\left\{\sqrt{a b c_{1} d_{1} \mathrm{e}_{1} f_{1}}(\mathrm{a} \partial \sigma-\mathrm{b} \partial \varpi)-\sqrt{\mathrm{a}_{1} \mathrm{~b}_{1} \operatorname{cdef}}\left(\mathrm{a}_{1} \partial \sigma-\mathrm{b}_{1} \partial \varpi\right)\right\}:
$$

but I shall not in the sequel use this formula, or the notation $\partial v-b \partial u=\partial \sigma$ introduced for obtaining it.

\section{Expression for $A \partial A B-A B \partial A$.}

Starting from the equations $A=\Omega \sqrt{ } a$ and $A B=\Omega \sqrt{a b}$, we have

$$
A \partial A B-A B \partial A=\Omega^{2}\{\sqrt{ } a \partial \sqrt{a b}-\sqrt{a b} \partial \sqrt{ } a\},
$$

where the term in \{\} is

$$
\begin{aligned}
& =\sqrt{\mathrm{aa}_{1}}\left[\frac{1}{2} \sqrt{\mathrm{abfa}_{1} \mathrm{~b}_{1} \mathrm{f}_{1}}\left\{\partial v+\left(\frac{\mathrm{cde}+\mathrm{c}_{1} \mathrm{~d}_{1} \mathrm{e}_{1}}{\theta^{2}}-c-d-e+x+y\right) \partial u\right\}\right. \\
& \left.+\frac{1}{2} \sqrt{\operatorname{cdec}_{1} \mathrm{~d}_{1} \mathrm{e}_{1}}\left\{-\partial v-\left(\frac{a b f+\mathrm{a}_{1} \mathrm{~b}_{1} \mathrm{f}_{1}}{\theta^{2}}-a-b-f+x+y\right) \partial u\right\}\right] \\
& -\frac{\frac{1}{2}}{\theta^{2}}\left(\sqrt{a b f c_{1} d_{1} e_{1}}-\sqrt{a_{1} b_{1} f_{1} c d e}\right)\left[\sqrt{a_{b_{1}} c_{1} d_{1} \mathrm{e}_{1} f_{1}}(\partial v-x \partial u)-\sqrt{a_{1} b c d e f}(\partial v-y \partial u)\right] .
\end{aligned}
$$

To reduce this, I write $\partial v-a \partial u=\partial \varpi$, and therefore

$$
\partial v-x \partial u=\partial \varpi+\mathrm{a} \partial u, \quad \partial v-y \partial u=\partial \varpi+\mathrm{a}_{1} \partial u
$$

then for convenience multiplying by $2 \theta^{2}$, the term is

$$
\begin{gathered}
=\mathrm{aa}_{1} \sqrt{\mathrm{bfb}_{1} \mathrm{f}_{1}}\left\{\theta^{2} \partial \varpi+\left[(a-c-d-e+x+y) \theta^{2}+c d e+c_{1} \mathrm{~d}_{1} \mathrm{e}_{1}\right] \partial u\right\} \\
+\sqrt{\operatorname{acdea}_{1} \mathrm{c}_{1} \mathrm{~d}_{1} \mathrm{e}_{1}}\left\{-\theta^{2} \partial \varpi+\left[\quad(b+f-x-y) \theta^{2}-\mathrm{abf}-\mathrm{a}_{1} \mathrm{~b}_{1} \mathrm{f}_{1}\right] \partial u\right\} \\
-\left(\sqrt{\mathrm{abfc}_{1} \mathrm{~d}_{1} \mathrm{e}_{1}}-\sqrt{\left.\mathrm{a}_{1} \mathrm{~b}_{1} \mathrm{f}_{1} \mathrm{cde}\right)}\left\{\sqrt{\mathrm{ab}_{1} \mathrm{c}_{1} \mathrm{~d}_{1} \mathrm{e}_{1} \mathrm{f}_{1}}(\partial \varpi+\mathrm{a} \partial u)-\sqrt{\mathrm{a}_{1} \mathrm{bcdef}}\left(\partial \varpi+\mathrm{a}_{1} \partial u\right)\right\} .\right.
\end{gathered}
$$

The last line hereof is

$$
\begin{array}{rr}
= & \sqrt{\mathrm{bfb}_{1} \mathrm{f}_{1}}\left\{-\mathrm{ac}_{1} \mathrm{~d}_{1} \mathrm{e}_{1}(\partial \varpi+\mathrm{a} \partial u)-\mathrm{a}_{1} \operatorname{cde}\left(\partial \varpi+\mathrm{a}_{1} \partial u\right)\right\} \\
+\sqrt{\operatorname{acdea}_{1} \mathrm{c}_{1} \mathrm{~d}_{1} \mathrm{e}_{1}}\{ & \left.\mathrm{b}_{1} \mathrm{f}_{1}(\partial \varpi+\mathrm{a} \partial u)+\quad \mathrm{bf}\left(\partial \varpi+\mathrm{a}_{1} \partial u\right)\right\} .
\end{array}
$$

Hence we have first, a term in $\sqrt{\text { acdea }_{1} c_{1} d_{1} e_{1}}$, the coefficient of which is

$$
=-\theta^{2} \partial \varpi+\left[(b+f-x-y) \theta^{2}-\mathrm{abf}-\mathrm{a}_{1} \mathrm{~b}_{1} \mathrm{f}_{1}\right] \partial u+\mathrm{b}_{1} \mathrm{f}_{1}(\partial \varpi+\mathrm{a} \partial u)+\mathrm{bf}\left(\partial \varpi+\mathrm{a}_{1} \partial u\right),
$$

viz. this is

$$
=\partial \varpi\left(-\theta^{2}+b_{1} f_{1}+b f\right)+\partial u\left[-\left(a-a_{1}\right)\left(b f-b_{1} f_{1}\right)+(b+f-x-y) \theta^{2}\right],
$$

where $\left(b-b_{1}\right)\left(f-f_{1}\right)=\theta^{2}$, that is, bf $+b_{1} f_{1}-\theta^{2}=b f_{1}+b_{1} f$, also

$$
\left(a-a_{1}\right)\left(b f-b_{1} f_{1}\right)=(b+f-x-y) \theta^{2},
$$

or the coefficient is $=\left(b f_{1}+b_{1} f\right) \partial \varpi$ : viz. the term in question is

$$
=\sqrt{\operatorname{acdea}_{1} c_{1} d_{1} e_{1}}\left(b f_{1}+b_{1} f\right) \partial \sigma .
$$


We have then, secondly, a term in $\sqrt{\mathrm{bfb}_{1} f_{1}}$, the coefficient of which is

viz. this is

$$
\begin{gathered}
=\mathrm{aa}_{1}\left\{\theta^{2} \partial \varpi+\left[(\alpha-c-d-e+x+y) \theta^{2}+c d e+c_{1} \mathrm{~d}_{1} \mathrm{e}_{1}\right] \partial u\right\} \\
-\mathrm{ac}_{1} \mathrm{~d}_{1} \mathrm{e}_{1}(\partial \varpi+\mathrm{a} \partial u)-\mathrm{a}_{1} \operatorname{cde}\left(\partial \varpi+\mathrm{a}_{1} \partial u\right),
\end{gathered}
$$

$=\left(a_{1} \theta^{2}-a_{1} d_{1} e_{1}-a_{1} c d e\right) \partial \sigma+\left[a_{1}(a-c-d-e+x+y) \theta^{2}+\left(a_{1} c d e-a_{1} d_{1} e_{1}\right)\left(a-a_{1}\right)\right] \partial u$.

We have $a-a_{1}=-\theta$; also $a_{1} c d e-a c_{1} d_{1} e_{1}$

$$
=\theta\left\{c d e-a(c d+c e+d e)+(c+d+e)[a(x+y)-x y]-a\left(x^{2}+x y+y^{2}\right)+x y(x+y)\right\},
$$

where the coefficient of $\theta$ is

$$
=-(a-c)(a-d)(a-e)-(c+d+e)\left[a^{2}-a(x+y)+x y\right]+(a+x+y)\left[a^{2}-a(x+y)+x y\right],
$$

viz. it is

$$
=-(\alpha-c)(a-d)(a-e)+\mathrm{aa}_{1}(\alpha-c-d-e+x+y) .
$$

Hence the coefficient in question is

$$
=\left(\mathrm{aa}_{1} \theta^{2}-\mathrm{ac}_{1} \mathrm{~d}_{1} \mathrm{e}_{1}-\mathrm{a}_{1} \mathrm{cde}\right) \partial \varpi+(a-c)(a-d)(a-e) \theta^{2} \partial u,
$$

and the second term is $=\sqrt{b_{b} b_{1} f_{1}}$, multiplied by this coefficient.

Hence, observing that the whole has to be multiplied by $\frac{\frac{1}{2}}{\theta^{2}} \Omega^{2}$, we find

$$
\begin{gathered}
A \partial A B-A B \partial A=\frac{\frac{1}{2}}{\theta^{2}} \Omega^{2}\left\{\sqrt{\operatorname{acdea}_{1} \mathrm{c}_{1} \mathrm{~d}_{1} \mathrm{e}_{1}}\left(\mathrm{bf}_{1}+\mathrm{b}_{1} \mathrm{f}\right) \partial \varpi\right. \\
\left.+\sqrt{\mathrm{bfb}_{1} \mathrm{f}_{1}}\left[\left(\mathrm{aa}_{1} \theta^{2}-\mathrm{ac}_{1} \mathrm{~d}_{1} \mathrm{e}_{1}-\mathrm{a}_{1} \mathrm{cde}\right) \partial \varpi+(a-c)(a-d)(a-e) \theta^{2} \partial u\right]\right\}
\end{gathered}
$$

where I retain $\partial \omega$ in place of its value, $=\partial v-\alpha \partial u$.

This is a sum of products of the set $b b_{1} f_{1}$ : we, in fact, have

$$
\begin{aligned}
& \sqrt{a c} \sqrt{d e}=\frac{1}{\theta^{2}}\left\{\left(b f_{1}+b_{1} f\right) \sqrt{a c d e a_{1} c_{1} d_{1} e_{1}}-\left(\operatorname{acd}_{1} e_{1}+a_{1} c_{1} d e\right) \sqrt{b f b_{1} f_{1}}\right\}, \\
& \sqrt{a d} \sqrt{c e}=\eta\left\{\quad, \quad, \quad-\left(\operatorname{adc}_{1} \mathrm{e}_{1}+\mathrm{a}_{1} \mathrm{~d}_{1} \mathrm{ce}\right) \quad \#\right\}, \\
& \sqrt{a e} \sqrt{c d}="\left\{\quad, \quad-\left(\operatorname{aec}_{1} \mathrm{~d}_{1}+\mathrm{a}_{1} \mathrm{e}_{1} c \mathrm{~d}\right) \quad \text { " }\right\} \text {, } \\
& \sqrt{ } b \sqrt{ } f="\left\{+\theta^{2}, "\right\},
\end{aligned}
$$

and selecting any two of these, for instance the first and the fourth, the coefficient of $\frac{\frac{1}{2}}{\theta^{2}} \Omega^{2}$ is at once seen to be of the form $\partial \varpi \sqrt{a c} \sqrt{d e}+K \sqrt{ } b \sqrt{ } f$; and for the determination of $K$, we have

$$
\left(-\operatorname{acd}_{1} \mathrm{e}_{1}-\mathrm{a}_{1} \mathrm{c}_{1} \mathrm{de}\right) \partial \varpi+K \theta^{2}=\left(\mathrm{aa}_{1} \theta^{2}-\mathrm{ac}_{1} \mathrm{~d}_{1} \mathrm{e}_{1}-\mathrm{a}_{1} \mathrm{cde}\right) \partial \varpi+(a-c)(a-d)(\alpha-e) \theta^{2} \partial u,
$$

viz. this gives

$$
K \theta^{2}=\left\{\mathrm{aa}_{1} \theta^{2}+\left(\mathrm{c}-\mathrm{c}_{1}\right)\left(\mathrm{ad}_{1} \mathrm{e}_{1}-\mathrm{a}_{1} \mathrm{de}\right)\right\} \partial \varpi+(a-c)(a-d)(a-e) \theta^{2} \partial u .
$$


We then have

$$
\left(c-c_{1}\right)\left(a d_{1} e_{1}-a_{1} d e\right)=\theta^{2}\left\{-a_{1}+(a-d)(a-e)\right\},
$$

and the whole equation divides by $\theta^{2}$; substituting for $\partial \varpi$ its value, we find

$$
K=(a-d)(a-e)(\partial v-c \partial u) .
$$

\section{Expression for $A C \partial A B-A B \partial A C$.}

Starting in like manner from the equations $A B=\Omega \sqrt{a b}, A C=\Omega \sqrt{a c}$, we have

multiplied by

$$
A C \partial A B-A B \partial A C=\frac{\frac{1}{2}}{\theta} \Omega^{2}
$$

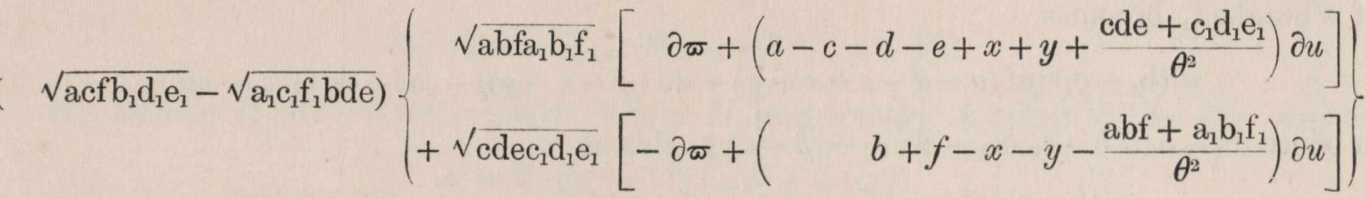

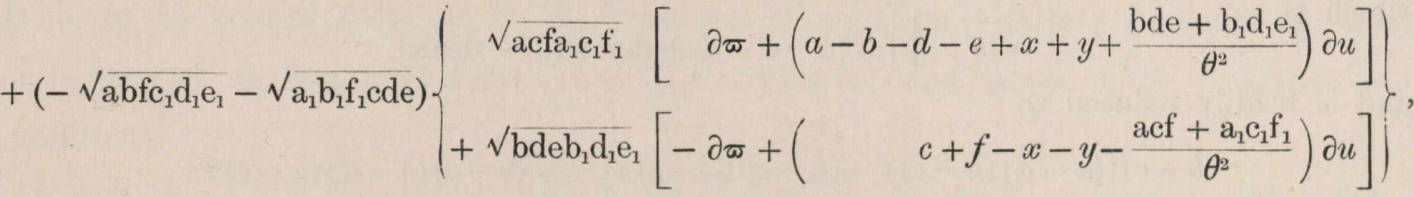

which, omitting the factor $\frac{\frac{1}{2}}{\theta} \Omega^{2}$, is

$$
\begin{aligned}
& =\left\{a b_{1} \sqrt{b c a_{1} d_{1} e_{1} f_{1}}-a_{1} f_{1} b \sqrt{b_{1} c_{1} a d e f}\right\}\left[\partial \varpi+\left(a-c-d-e+x+y+\frac{\text { cde }+c_{1} d_{1} e_{1}}{\theta^{2}}\right) \partial u\right] \\
& +\left\{\operatorname{cd}_{1} \mathrm{e}_{1} \sqrt{\mathrm{b}_{1} \mathrm{c}_{1} \mathrm{adef}}-c_{1} \mathrm{de} \sqrt{\left.\mathrm{bca}_{1} \mathrm{~d}_{1} \mathrm{e}_{1} \mathrm{f}_{1}\right\}}\left[-\partial \boldsymbol{t}+\left(\quad b+f-x-y-\frac{a b f+\mathrm{a}_{1} \mathrm{~b}_{1} \mathrm{f}_{1}}{\theta^{2}}\right) \partial u\right]\right. \\
& +\left\{-\operatorname{afc}_{1} \sqrt{\mathrm{bca}_{1} \mathrm{~d}_{1} \mathrm{e}_{1} \mathrm{f}_{1}}+\mathrm{a}_{1} \mathrm{f}_{1} \mathrm{c} \sqrt{\mathrm{b}_{1} \mathrm{c}_{1} \mathrm{adef}}\right\}\left[\partial \varpi+\left(a-b-d-e+x+y+\frac{\mathrm{bde}+\mathrm{b}_{1} \mathrm{~d}_{1} \mathrm{e}_{1}}{\theta^{2}}\right) \partial u\right] \\
& +\left\{- \text { bde }_{1} \sqrt{b_{1} c_{1} a d e f}+b_{1} \operatorname{de} \sqrt{b_{c c a} d_{1} e_{1} f_{1}}\right\}\left[-\partial \varpi+\left(c+f-x-y-\frac{a c f+a_{1} c_{1} f_{1}}{\theta^{2}}\right) \partial u\right] ;
\end{aligned}
$$

and here the whole coefficient of $\partial \omega^{*}$ is

$$
=\left(b_{1}-c_{1}\right)(a f-d e) \sqrt{b c a_{1} d_{1} e_{1} f_{1}}-(b-c)\left(a_{1} f_{1}-d_{1} e_{1}\right) \sqrt{b_{1} c_{1} a d e f},
$$

viz. observing that $\mathrm{b}_{1}-\mathrm{c}_{1}=\mathrm{b}-\mathrm{c}=b-c$, this is

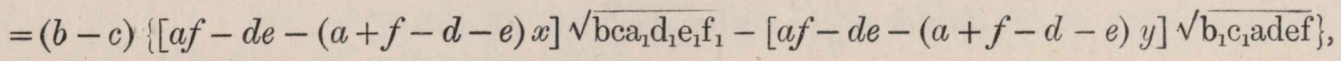

or, what is the same thing, it is

$$
\begin{array}{r}
=(b-c)\left\{[-(a-d)(a-e)+(a+f-d-e) \mathrm{a}] \sqrt{\mathrm{bca}_{1} \mathrm{~d}_{1} \mathrm{e}_{1} \mathrm{f}_{1}}\right. \\
\left.-\left[-(a-d)(a-e)+(a+f-d-e) \mathrm{a}_{1}\right] \sqrt{\mathrm{b}_{1} \mathrm{c}_{1} \mathrm{adef}}\right\} .
\end{array}
$$


The coefficient of $\partial u$ contains the factor $\sqrt{\text { bca }_{1} \mathrm{~d}_{1} \mathrm{e}_{1} \mathrm{f}_{1}}$, multiplied by

$$
\begin{aligned}
& \mathrm{afb}_{1}\left(a-c-d-e+x+y+\frac{c d e+\mathrm{c}_{1} \mathrm{~d}_{1} \mathrm{e}_{1}}{\theta^{2}}\right) \\
& -c_{1} \operatorname{de}\left(\quad b+f-x-y-\frac{a b f+a_{1} b_{1} f_{1}}{\theta^{2}}\right) \\
& -\operatorname{afc}_{1}\left(a-b-d-e+x+y+\frac{b d e+b_{1} d_{1} e_{1}}{\theta^{2}}\right) \\
& +b_{1} \operatorname{de}\left(\quad c+f-x-y-\frac{a c f+a_{1} c_{1} f_{1}}{\theta^{2}}\right) ;
\end{aligned}
$$

here the terms divided by $\theta^{2}$ destroy each other, and the expression of the coefficient of $\sqrt{\text { bca }_{1} d_{1} e_{1} f_{1}}$ becomes

$$
=\left(b_{1}-c_{1}\right)[a f(a-d-e+x+y)+\operatorname{de}(f-x-y)]+(a f-d e)\left(b c_{1}-c b_{1}\right),
$$

or since $\mathrm{b}_{1}-\mathrm{c}_{1}=b-c, b \mathrm{c}_{1}-c \mathrm{~b}_{1}=-(b-c) y$, this is

which is

$$
=(b-c)[\operatorname{af}(a-d-e+x+y)+\operatorname{de}(f-x-y)-(\operatorname{af}-\mathrm{de}) y],
$$

$$
=(b-c)[\operatorname{af}(a-d-e)+\operatorname{def}+(\operatorname{af}-\operatorname{de}) x],
$$

and is readily reduced to

$$
(b-c)[(a-d)(a-e) f-(a-d)(a-e) x],=(b-c)(a-d)(a-e) \mathrm{f}:
$$

viz. the coefficient of $\partial u$ contains the term $(b-c)(a-d)(a-e) \mathrm{f} \sqrt{\mathrm{bca}_{1} \mathrm{~d}_{1} \mathrm{e}_{1} \mathrm{f}_{1}}$. There is

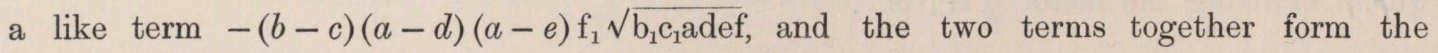
whole coefficient of $\partial u$.

Hence, restoring the outside factor $\frac{\frac{1}{2}}{\theta} \Omega^{2}$, we have

$A C \partial A B-A B \partial A C$

$$
\begin{aligned}
= & =\frac{1}{\theta} \Omega^{2}(b-c)\left[\left\{[-(a-d)(a-e)+(a+f-d-e) a] \sqrt{\mathrm{bca}_{1} \mathrm{~d}_{1} \mathrm{e}_{1} \mathrm{f}_{1}}\right.\right. \\
& \left.-\left[-(a-d)(a-e)+(a+f-d-e) \mathrm{a}_{1}\right] \sqrt{\mathrm{b}_{1} \mathrm{c}_{1} \mathrm{adef}}\right\} \partial \sigma \\
& \left.+(a-d)(a-e)\left\{\mathrm{f} \sqrt{\mathrm{bca}_{1} \mathrm{~d}_{1} \mathrm{e}_{1} \mathrm{f}_{1}}-\mathrm{f}_{1} \sqrt{\mathrm{b}_{1} \mathrm{c}_{1} \text { adef }}\right\} \partial u\right]
\end{aligned}
$$

where, as before, I retain $\partial \varpi$ instead of its value $=\partial v-a \partial u$. This is a sum of products of the set bc: the products, in fact, are

$$
\begin{aligned}
& \sqrt{ } a \sqrt{d e}=\frac{1}{\theta}\left\{-a \sqrt{b_{c a} d_{1} \mathrm{e}_{1} \mathrm{f}_{1}}+a_{1} \sqrt{\mathrm{b}_{1} \mathrm{c}_{1} \mathrm{adef}}\right\}, \\
& \sqrt{ } d \sqrt{a e}=,\left\{-\mathrm{d} \quad, \quad+\mathrm{d}_{1} \quad, \quad\right\}, \\
& \sqrt{e} \sqrt{a d}=,\left\{-\mathrm{e} \quad, \quad+\mathrm{e}_{1} \quad,\right\} \text {, } \\
& \sqrt{ } f \sqrt{b c}=,\left\{+\mathrm{f} \quad, \quad-\mathrm{f}_{1} \quad, \quad\right\},
\end{aligned}
$$


whence, observing that $\mathrm{a}-\mathrm{f}=\mathrm{a}_{1}-\mathrm{f}_{1}=a-f$, we have

$$
\sqrt{ } a \sqrt{d e}+\sqrt{ } f \sqrt{b c}=-\frac{a-f}{\theta}\left\{\sqrt{b_{c a} d_{1} \mathrm{e}_{1} f_{1}}-\sqrt{b_{1} c_{1} a d e f}\right\}:
$$

it is clear that the term in question is at once expressible as a sum formed with the products $\sqrt{ } a \sqrt{d e}$ and $\sqrt{ } f \sqrt{b c}$.

It is to be remarked that there are 15 expressions such as $A \partial B-B \partial A$, and 45 expressions such as $A C \partial A B-A B \partial A C$; and that each of these $(15+45=) 60$ expressions is a sum of products of a set such as ab: and that there are also 60 expressions of the form $A \partial A B-A B \partial A$, and that each of these is a sum of products of a set such as $a b a_{1} b_{1}$.

$$
\text { Expression of } \Omega \partial^{2} \Omega-(\partial \Omega)^{2}, \quad=\frac{1}{4} M \Omega^{2} \text {. }
$$

We assume $\Omega \partial^{2} \Omega-(\partial \Omega)^{2}=\frac{1}{4} M \Omega^{2}$, where $M$ is a quadric function of $\partial u$, $\partial v$; suppose

$$
M=\mathfrak{A}(\partial u)^{2}+2 \mathfrak{B} \partial u \partial v+\left(5(\partial v)^{2} .\right.
$$

It is to be noticed that the $\mathfrak{A}, \mathfrak{B},(\mathcal{E}$ are not all of them arbitrary functions of $(x, y)$ or $(u, v)$; we, in fact, have $\frac{1}{4} M=\frac{\partial^{2} \Omega}{\Omega}-\frac{(\partial \Omega)^{2}}{\Omega^{2}}=\partial^{2} \log \Omega ;$ and hence $\mathfrak{A}, \mathfrak{B},(5$ satisfy the conditions

$$
\frac{d \mathfrak{A}}{d v}=\frac{d \mathfrak{B}}{d u}, \quad \frac{d \mathfrak{B}}{d v}=\frac{d \mathfrak{E}}{d u} .
$$

Taking $\mathfrak{A}, \mathfrak{B}, \mathfrak{C}$ as functions of $x, y$, these become

$$
\begin{aligned}
& \left(\frac{d \mathfrak{A}}{d x}+y \frac{d \mathfrak{B}}{d x}\right) \sqrt{X}=\left(\frac{d \mathfrak{A}}{d y}+x \frac{d \mathfrak{B}}{d y}\right) \sqrt{Y}, \\
& \left(\frac{d \mathfrak{B}}{d x}+y \frac{d(\widetilde{S}}{d x}\right) \sqrt{X}=\left(\frac{d \mathfrak{B}}{d y}+x \frac{d \mathfrak{(}}{d y}\right) \sqrt{Y} .
\end{aligned}
$$

Putting for the moment

$$
\begin{array}{lll}
\lambda=a+b+c, & \rho=d+e+f, & p=\lambda+\rho, \\
\mu=a b+a c+b c, & \sigma=d e+d f+e f, & q=\mu+\sigma, \\
\nu=a b c, & \tau=d e f, & r=\nu+\tau,
\end{array}
$$

I found it convenient to assume

$$
5=-2\left(x^{2}+x y+y^{2}\right)+p(x+y)
$$

where observe that $p,=a+b+c+d+e+f$, is symmetrical in regard to the constants $a, b, c, d, e, f$. And then, $(5$ having this value, there exists (as is seen at once) a value of $\mathfrak{B},=2\left(x^{2} y+x y^{2}\right)-p x y$, for which

$$
\frac{d \mathfrak{B}}{d x}+y \frac{d \mathfrak{E}}{d x}=0, \quad \frac{d \mathfrak{B}}{d y}+x \frac{d \mathfrak{E}}{d y}=0,
$$

and which thus satisfies the second of the above-mentioned conditions.

C. $\mathrm{X}$. 
Assuming now

$$
\mathfrak{A}=-2 x^{2} y^{2}+q x y-r(x+y)-\mu \sigma+\Theta,
$$

where $\Theta$ has to be determined so as that the first of the same conditions may be also satisfied, then substituting this value of $\mathfrak{A}$, we have

that is,

$$
\left(2 y^{3}-p y^{2}+q y-r+\frac{d \Theta}{d x}\right) \sqrt{X}=\left(2 x^{3}-p x^{2}+q x-r+\frac{d \Theta}{d y}\right) \sqrt{Y}
$$

$$
\left(-\mathrm{a}_{1} \mathrm{~b}_{1} \mathrm{c}_{1}-\mathrm{d}_{1} \mathrm{e}_{1} \mathrm{f}_{1}+\frac{d \Theta}{d x}\right) \sqrt{X}=\left(-\mathrm{abc}-\operatorname{def}+\frac{d \Theta}{d y}\right) \sqrt{\bar{Y}}
$$

viz. in the terms independent of $\Theta$ writing for $\sqrt{ } X, \sqrt{ } Y$ their values, this is

$$
(a b c+d e f) \sqrt{a_{1} b_{1} c_{1} d_{1} e_{1} f_{1}}-\left(a_{1} b_{1} c_{1}+d_{1} e_{1} f_{1}\right) \sqrt{a b c d e f}+\frac{d \Theta}{d x} \sqrt{X}-\frac{d \Theta}{d y} \sqrt{Y}=0,
$$

or, what is the same thing,

$$
-\left(\sqrt{\operatorname{abca}_{1} b_{1} c_{1}}-\sqrt{\operatorname{defd}_{1} e_{1} f_{1}}\right)\left(\sqrt{\operatorname{defa}_{1} b_{1} c_{1}}-\sqrt{d_{1} e_{1} f_{1} a b c}\right)+\frac{d \Theta}{d x} \sqrt{X}-\frac{d \Theta}{d y} \sqrt{Y}=0 .
$$

But treating $\Theta$ as a function of $u$ and $v$, we have

also

$$
\frac{d \Theta}{d v}=\frac{d \Theta}{d x} \frac{d x}{d v}+\frac{d \Theta}{d y} \frac{d y}{d v}=\frac{1}{\theta}\left(\frac{d \Theta}{d x} \sqrt{X}-\frac{d \Theta}{d y} \sqrt{Y}\right):
$$

$$
\sqrt{d e}=\frac{1}{\theta}\left(\sqrt{\text { defa }_{1} b_{1} c_{1}}-\sqrt{d_{1} e_{1} f_{1} a b c}\right)
$$

and we thus reduce the equation to

$$
-\left(\sqrt{\mathrm{abca}_{1} \mathrm{~b}_{1} \mathrm{c}_{1}}-\sqrt{\operatorname{defd}_{1} \mathrm{e}_{1} \mathrm{f}_{1}}\right) \sqrt{d e}+\frac{d \Theta}{d v}=0 .
$$

But, referring to the expression for $\partial \sqrt{a b}$, we have, by a mere interchange of letters,

$$
\frac{d}{d v} \sqrt{d e}=-\frac{1}{2}\left(\sqrt{\operatorname{abca}_{1} \mathrm{~b}_{1} \mathrm{c}_{1}}-\sqrt{\operatorname{defd}_{1} \mathrm{e}_{1} \mathrm{f}_{1}}\right)
$$

and the formula thus becomes

consequently

$$
2 \sqrt{d e} \frac{d}{d v} \sqrt{d e}+\frac{d \Theta}{d v}=0
$$

$$
\Theta=-(\sqrt{d e})^{2}=-\frac{1}{\theta^{2}}\left(\operatorname{abcd}_{1} \mathrm{e}_{1} \mathrm{f}_{1}+\mathrm{a}_{1} \mathrm{~b}_{1} \mathrm{c}_{1} \operatorname{def}-2 \sqrt{X Y}\right),
$$

and the value of $\mathfrak{A}$ thus is

$$
\mathfrak{A}=\frac{1}{\theta^{2}}\left\{-\operatorname{abcd}_{1} \mathrm{e}_{1} \mathrm{f}_{1}-\mathrm{a}_{1} \mathrm{~b}_{1} \mathrm{c}_{1} \mathrm{def}+\theta^{2}\left(-2 x^{2} y^{2}+q x y-r(x+y)-\mu \sigma\right)\right\}+\frac{2}{\theta^{2}} \sqrt{X Y},
$$

or, as this may be written,

$$
\mathfrak{A}=\frac{1}{\theta^{2}}\left(a b c-a_{1} b_{1} c_{1}\right)\left(d e f-d_{1} e_{1} f_{1}\right)-2 x^{2} y^{2}+q x y-r(x+y)-\mu \sigma-\frac{1}{\theta^{2}}(\sqrt{X}-\sqrt{Y})^{2} .
$$


Here

and similarly

$$
\begin{aligned}
\operatorname{abc}-\mathrm{a}_{1} \mathrm{~b}_{1} \mathrm{c}_{1} & =\left(\nu-\mu x+\lambda x^{2}-x^{3}\right)-\left(\nu-\mu y+\lambda y^{2}-y^{3}\right) \\
& =\theta\left[-\mu+\lambda(x+y)-\left(x^{2}+x y+y^{2}\right)\right], \\
\operatorname{def}-\mathrm{d}_{2} \mathrm{e}_{1} \mathrm{f}_{1} & =\theta\left[-\sigma+\rho(x+y)-\left(x^{2}+x y+y^{2}\right)\right] ;
\end{aligned}
$$

the expression of $\mathfrak{A}$ contains therefore the terms

$$
\left[\mu-\lambda(x+y)+x^{2}+x y+y^{2}\right]\left[\sigma-\rho(x+y)+x^{2}+x y+y^{2}\right]-\mu \sigma-r(x+y)+q x y-2 x^{2} y^{2},
$$

viz. for $r, q$ substituting their values $\nu+\tau, \mu+\rho$, these terms are

$$
\begin{aligned}
= & -(\mu \rho+\sigma \lambda+\nu+\tau)(x+y)+(\mu+\sigma+\lambda \rho)(x+y)^{2} \\
& -(\lambda+\rho)(x+y)\left(x^{2}+x y+y^{2}\right)+\left(x^{2}+x y+y^{2}\right)^{2}-2 x^{2} y^{2} .
\end{aligned}
$$

The coefficients $\mu \rho+\sigma \lambda+\nu+\tau, \mu+\sigma+\lambda \rho, \lambda+\rho$ are, in fact, symmetrical functions of $a, b, c, d, e, f$, viz. writing

that is,

$$
\begin{aligned}
X & =a-x \cdot b-x \cdot c-x \cdot d-x \cdot e-x \cdot f-x, \\
& =\mathrm{A}-\mathrm{B} x+\mathrm{C} x^{2}-\mathrm{D} x^{3}+\mathrm{E} x^{4}-\mathrm{F} x^{5}+x^{6},
\end{aligned}
$$

$$
\mathbf{A}=a b c d e f, \quad \mathbf{B}=\Sigma a b c d e, \quad \mathbf{C}=\Sigma a b c d, \quad \mathbf{D}=\Sigma a b c, \quad \mathbf{E}=\Sigma a b, \quad \mathbf{F}=\Sigma a,
$$

$(\mathrm{F}=a+b+c+d+e+f$, which has in fact previously been called $p$ ), we have

and the terms are

$$
\mu \rho+\sigma \lambda+\nu+\tau=\mathrm{D}, \quad \mu+\sigma+\lambda \rho=\mathrm{E}, \quad \lambda+\rho=\mathrm{F},
$$

$$
=-(x+y)\left\{\mathrm{D}-\mathrm{E}(x+y)+\mathrm{F}\left(x^{2}+x y+y^{2}\right)\right\}+x^{4}+2 x^{3} y+x^{2} y^{2}+2 x y^{3}+y^{4} ;
$$

viz. we have

$$
\begin{aligned}
\mathfrak{A}= & -\frac{1}{\theta^{2}}(\sqrt{X}-\sqrt{Y})^{2} \\
& -(x+y)\left\{\mathrm{D}-\mathrm{E}(x+y)+\mathrm{F}\left(x^{2}+x y+y^{2}\right)\right\}+\left(x^{4}+2 x^{3} y+x^{2} y^{2}+2 x y^{3}+y^{4}\right) .
\end{aligned}
$$

To this I join the foregoing values of $\mathfrak{B}, \sqrt{5}$; viz. writing $\mathrm{F}$ in place of $p$, these are

$$
\begin{aligned}
& \mathfrak{B}=-\mathbf{F} x y+2\left(x^{2} y+x y^{2}\right), \\
& \mathfrak{E}=\mathrm{F}(x+y)-2\left(x^{2}+x y+y^{2}\right),
\end{aligned}
$$

where it will be noticed that the values of $\mathfrak{A}, \mathfrak{B}$, $\mathfrak{E}$ are all of them symmetrical in regard to the constants $a, b, c, d, e, f$.

I recall the original form of $\mathfrak{A}$, viz. this was

$$
\begin{aligned}
\mathfrak{A}= & -\mu \sigma-r(x+y)+q x y-2 x^{2} y^{2}-(\sqrt{d e})^{2} \\
= & -(a b+a c+b c)(d e+d f+e f)-(a b c+d e f)(x+y) \\
& +(a b+a c+b c+d e+d f+e f) x y-2 x^{2} y^{2}-(\sqrt{d e})^{2} \\
= & \mathfrak{A}_{0}-(\sqrt{d e})^{2},
\end{aligned}
$$


suppose; and $\mathfrak{A}, \mathfrak{B}$, $\mathfrak{C}$ denoting as above, we have

$$
M=\mathfrak{A}(\partial u)^{2}+2 \mathfrak{B} \partial u \partial v+\left(\complement(\partial v)^{2}, \quad \Omega \partial^{2} \Omega-(\partial \Omega)^{2}=\frac{1}{4} M \Omega^{2} .\right.
$$

For the subsequent calculation of $A \partial^{2} A-(\partial A)^{2}$, it is convenient to transform this expression by introducing therein $\partial \varpi$ in place of $\partial v$, and $a, a_{1}$ in place of $x, y$. We have

suppose, where

$$
\begin{aligned}
M & =\left\{\mathfrak{F}_{0}-(\sqrt{d e})^{2}\right\}(\partial u)^{2}+2 \mathfrak{B} \partial u(\partial \varpi+a \partial u)+\left(\complement(\partial \varpi+a \partial u)^{2}\right. \\
& =\left\{\mathfrak{F}_{0}^{\prime}-(\sqrt{d e})^{2}\right\}(\partial u)^{2}+2 \mathfrak{B}^{\prime} \partial u \partial \varpi+\left(\mathcal{\zeta}^{\prime}(\partial \varpi)^{2},\right.
\end{aligned}
$$

Writing

we find

$$
\begin{aligned}
& \mathfrak{\complement}^{\prime}=\mathfrak{\complement} \\
& \mathfrak{B}^{\prime}=\mathfrak{B}+a \mathfrak{\complement}, \\
& \mathfrak{A}_{0}^{\prime}=\mathfrak{A}_{0}+2 a \mathfrak{B}+a^{2} \mathfrak{\complement} .
\end{aligned}
$$

$$
\begin{aligned}
\mathfrak{C}= & -6 a^{2}+2 a \mathrm{~F}+(6 a-\mathrm{F})\left(\mathrm{a}+\mathrm{a}_{1}\right)-2\left(\mathrm{a}^{2}+a \mathrm{a}_{1}+\mathrm{a}_{1}^{2}\right) \\
\mathfrak{B}= & 4 a^{3}-a^{2} \mathrm{~F}+\left(-6 a^{2}+a \mathrm{~F}\right)\left(\mathrm{a}+\mathrm{a}_{1}\right)+2 a\left(\mathrm{a}^{2}+\mathrm{a}_{1}^{2}\right)+(8 a-\mathrm{F}) \mathrm{aa}_{1}-2\left(\mathrm{a}^{2} \mathrm{a}_{1}+\mathrm{aa}_{1}^{2}\right) \\
\mathfrak{N}_{0}= & -(a b+a c+b c)(d e+d f+e f) \\
& -(a b c+d e f)\left(2 a-a-\mathrm{a}_{1}\right) \\
& +(a b+a c+b c+d e+d f+e f)\left[a^{2}-a\left(\mathrm{a}+\mathrm{a}_{1}\right)+a a_{1}\right] \\
& -2\left[a^{2}-a\left(a+a_{1}\right)+a a_{1}\right]^{2},
\end{aligned}
$$

the developed value of which is

$$
\begin{aligned}
= & -2 a^{4}+a^{3}(b+c)+a^{2}(-b c+d e+d f+e f) \\
& +a\{-2 d e f-(b+c)(d e+d f+e f)\}-b c(d e+d f+e f) \\
& +\left\{4 a^{3}-a^{2}(b+c)-a(d e+d f+e f)+d e f\right\}\left(a+a_{1}\right) \\
& -2 a^{2}\left(a^{2}+a_{1}^{2}\right)+\left\{-8 a^{2}+a(b+c)+b c+d e+d f+e f\right\} a a_{1} \\
& +4 a\left(a^{2} a_{1}+a a_{1}^{2}\right) \\
& -2 a^{2} a_{1}^{2},
\end{aligned}
$$

and thence without difficulty

$$
\begin{aligned}
\delta^{\prime}= & -6 a^{2}+2 a \mathrm{~F}+(6 a-\mathrm{F})\left(\mathrm{a}+\mathrm{a}_{1}\right)-2\left(\mathrm{a}^{2}+\mathrm{aa}_{1}+\mathrm{a}_{1}^{2}\right) \\
\mathfrak{B}^{\prime}= & -8 a^{3}+a^{2} \mathrm{~F}+(6 a-\mathrm{F}) \mathrm{aa}_{1}-2\left(\mathrm{a}^{2} \mathrm{a}_{1}+\mathrm{aa}_{1}^{2}\right) \\
\mathfrak{A}_{0}^{\prime}= & a^{3}(b+c)+a^{2}(-b c+d e+d f+e f)+a\{-2 d e f-(b+c)(d e+d f+e f)\}-b c(d e+d f+e f) \\
& +\left\{-a^{3}+a^{2}(d+e+f)-a(d e+d f+e f)+d e f\right\}\left(\mathrm{a}+\mathrm{a}_{1}\right) \\
& +\left\{4 a^{2}+a(-b-c-2 d-2 e-2 f)+b c+d e+d f+e f\right\} \mathrm{aa}_{1} \\
& -2 \mathrm{a}^{2} \mathrm{a}_{1}{ }^{2}
\end{aligned}
$$

which are the required values. 
Expression for $A \partial^{2} A-(\partial A)^{2}$ : several subheadings.

Writing for shortness $A \partial^{2} A-(\partial A)^{2}=\Delta A$, as before, and so in other cases: then in general $\Delta P Q=P^{2} \Delta Q+Q^{2} \Delta P$, and thence $\Delta P^{2}=2 P^{2} \Delta P$ or $\Delta \sqrt{ } P=\frac{\frac{1}{2}}{P} \Delta P$. Hence starting from $A=\Omega \sqrt{ } a=\Omega \sqrt{\mathrm{aa}_{1}}$, we have

where

$$
\Delta A=\Delta \Omega \sqrt{\mathrm{aa}_{1}}=\mathrm{aa}_{1} \Delta \Omega+\frac{\frac{1}{2} \Omega^{2}}{\mathrm{aa}_{1}}\left(\mathrm{a}^{2} \Delta \mathrm{a}_{1}+\mathrm{a}_{1}{ }^{2} \Delta \mathrm{a}\right)
$$

$$
\Delta \mathrm{a}=\mathrm{a} \partial^{2} \mathrm{a}-(\partial \mathrm{a})^{2}=-\mathrm{a} \partial^{2} x-(\partial x)^{2}, \Delta \mathrm{a}_{1}=-\mathrm{a} \partial^{2} y-(\partial y)^{2}
$$

Hence writing

we have

$$
\Delta \Omega=\frac{1}{4} M \Omega^{2}
$$

But we have

$$
\frac{1}{\Omega^{2}} \Delta A=\frac{1}{4} \mathrm{aa}_{1} M-\frac{1}{2}\left(\mathrm{a}_{1} \partial^{2} x+\mathrm{a}^{2} x_{1}\right)-\frac{1}{2}\left\{\frac{\mathrm{a}_{1}}{\mathrm{a}}(\partial x)^{2}+\frac{\mathrm{a}}{\mathrm{a}_{1}}(\partial y)^{2}\right\} .
$$

$$
\partial x=\frac{\sqrt{ } X}{\theta}(\partial v-y \partial u), \quad \partial y=-\frac{\sqrt{ } \boldsymbol{Y}}{\theta}(\partial v-x \partial u)
$$

squaring the first of these and differentiating, we find

$$
2 \partial x \partial^{2} x=\left[\left(-\frac{2 X}{\theta^{3}}+\frac{X^{\prime}}{\theta^{2}}\right) \partial x+\frac{2 X}{\theta^{3}} \partial y\right](\partial v-y \partial u)^{2}-2 \partial y \partial u \frac{X}{\theta^{2}}(\partial v-y \partial u)
$$

where as regards $X$ the accent denotes differentiation as to $x$ (and further on, as regards $Y$, it denotes differentiation to $y$ ), viz. this is

$$
\begin{aligned}
& =\left[\left(-\frac{2 X}{\theta^{3}}+\frac{X^{\prime}}{\theta^{2}}\right) \partial x+\frac{2 X}{\theta^{3}} \partial y\right](\partial v-y \partial u)^{2}-2 \partial y \partial u \frac{X}{\theta^{2}}(\partial v-y \partial u) \\
& =\left(-\frac{2 X}{\theta^{3}}+\frac{X^{\prime}}{\theta^{2}}\right) \partial x(\partial v-y \partial u)^{2}+\frac{2 X}{\theta^{3}}(\partial v-y \partial u-\theta \partial u)(\partial v-y \partial u) \partial y
\end{aligned}
$$

where the second term is

which is

$$
\frac{2 X}{\theta^{3}}(\partial v-x \partial u)(\partial v-y \partial u) \partial y
$$

$$
=-\frac{2 \sqrt{X Y}}{\theta^{3}}(\partial v-x \partial u)^{2} \partial x:
$$

hence dividing by $2 \partial x$, the equation is

and similarly

$$
\partial^{2} x=\left(-\frac{X}{\theta^{3}}+\frac{X^{\prime}}{2 \theta^{2}}\right)(\partial v-y \partial u)^{2}-\frac{\sqrt{X Y}}{\theta^{3}}(\partial v-x \partial u)^{2}
$$

$$
\partial^{2} y=\frac{\sqrt{X Y}}{\theta^{3}}(\partial v-y \partial u)^{2}+\left(\frac{Y}{\theta^{3}}+\frac{Y^{\prime}}{2 \theta^{2}}\right)(\partial v-x \partial u)^{2}
$$

and we may in these values in place of $\partial v-y \partial u$ and $\partial v-x \partial u$ write $\partial \varpi+a_{1} \partial u$ and $\partial \varpi+\mathbf{a} \partial u$ respectively. 
Hence in $\frac{1}{\Omega^{2}} \Delta A$ the irrational part is

$$
\begin{aligned}
\frac{1}{2} & \frac{\sqrt{X Y}}{\theta^{3}}\left\{a_{1}(\partial \varpi+a \partial u)^{2}-a\left(\partial \varpi+a_{1} \partial u\right)^{2}\right\} \\
= & \frac{\frac{1}{2} \sqrt{X Y}}{\theta^{3}}\left(a_{1}-a\right)\left\{(\partial \varpi)^{2}-a a_{1}(\partial u)^{2}\right\}=\frac{\frac{1}{2} \sqrt{X Y}}{\theta^{2}}\left\{(\partial \varpi)^{2}-a a_{1}(\partial u)^{2}\right\}
\end{aligned}
$$

But we have

$$
(\sqrt{d e})^{2}=\frac{1}{\theta^{2}}\left\{\operatorname{abcd}_{1} \mathrm{e}_{1} \mathrm{f}_{1}+\mathrm{a}_{1} \mathrm{~b}_{1} \mathrm{c}_{1} \operatorname{def}-2 \sqrt{X Y}\right\}
$$

whence

$$
\frac{\sqrt{X Y}}{\theta^{2}}=\frac{\frac{1}{2}}{\theta^{2}}\left(\operatorname{abcd}_{1} e_{1} f_{1}+a_{1} b_{1} c_{1} d e f\right)-\frac{1}{2}(\sqrt{d e})^{2}
$$

and the term thus is

$$
\left[\frac{\frac{1}{4}}{\theta^{2}}\left(a b c d_{1} e_{1} f_{1}+a_{1} b_{1} c_{1} d e f\right)-\frac{1}{4}(\sqrt{d e})^{2}\right]\left\{(\partial \varpi)^{2}-a a_{1}(\partial u)^{2}\right\}
$$

Joining hereto the rational part of $\frac{1}{\Omega^{2}} \Delta A$, and multiplying the whole by 4 , we have

$$
\begin{aligned}
\frac{4}{\Omega^{2}} \Delta A=\mathrm{aa}_{1} M & +\left[\mathrm{a}_{1}\left(\frac{2 X}{\theta^{3}}-\frac{X^{\prime}}{\theta^{2}}\right)-\frac{2 \mathrm{a}_{1}}{\mathrm{a}} \frac{X}{\theta^{2}}\right]\left(\partial \varpi+\mathrm{a}_{1} \partial u\right)^{2} \\
+ & {\left[\mathrm{a}\left(-\frac{2 Y}{\theta^{3}}-\frac{Y^{\prime}}{\theta^{2}}\right)-\frac{2 \mathrm{a}}{\mathrm{a}_{1}} \frac{Y}{\theta^{2}}\right](\partial \varpi+\mathrm{a} \partial u)^{2} } \\
+ & {\left[\frac{1}{\theta^{2}}\left(\operatorname{abcd}_{1} \mathrm{e}_{1} \mathrm{f}_{1}+\mathrm{a}_{1} \mathrm{~b}_{1} \mathrm{c}_{1} \mathrm{def}\right)-(\sqrt{d e})^{2}\right]\left\{(\partial \varpi)^{2}-\mathrm{aa}_{1}(\partial u)^{2}\right\} }
\end{aligned}
$$

where $M$ has its foregoing value $=\left\{\mathfrak{U}_{0}^{\prime}-(\sqrt{d e})^{2}\right\}(\partial u)^{2}+2 \mathfrak{B}^{\prime} \partial u \partial \varpi+\left(\varsigma^{\prime}(\partial \varpi)^{2}\right.$.

\section{First step of the reduction.}

Writing bcdef $=U, \mathrm{~b}_{1} \mathrm{c}_{1} \mathrm{~d}_{1} \mathrm{e}_{1} \mathrm{f}_{1}=U_{1}$, then $X=\mathrm{a} U, Y=\mathrm{a}_{1} U_{1}$, and consequently

$$
X^{\prime}=-U+\mathrm{a} U^{\prime}, \quad Y^{\prime}=-U_{1}+\mathrm{a}_{1} U_{1}^{\prime},
$$

the accents in regard to $U, U_{1}$ denoting differentiations as to $x, y$ respectively: then

$$
\mathrm{a}_{1}\left(\frac{2 X}{\theta^{3}}-\frac{X^{\prime}}{\theta^{2}}\right)-\frac{2 \mathrm{a}_{1}}{\mathrm{a}} \frac{X}{\theta^{2}}=\mathrm{a}_{1}\left(\frac{2 \mathrm{a} U}{\theta^{3}}+\frac{U-\mathrm{a} U^{\prime}}{\theta^{2}}\right)-\frac{2 \mathrm{a}_{1}}{\mathrm{a}} \frac{\mathrm{a} U}{\theta^{2}}=\mathrm{aa}_{1}\left(\frac{2 U}{\theta^{3}}-\frac{U^{\prime}}{\theta^{2}}\right)-\frac{\mathrm{a}_{1} U}{\theta^{2}}:
$$

and similarly

$$
\mathrm{a}\left(-\frac{2 Y}{\theta^{3}}-\frac{Y^{\prime}}{\theta^{2}}\right)-\frac{2 \mathrm{a}}{\mathrm{a}_{1}} \frac{Y}{\theta^{2}}=\ldots . . . \quad . \quad . \quad . \quad .=\mathrm{aa}_{1}\left(-\frac{2 U_{1}}{\theta^{3}}-\frac{U_{1}^{\prime}}{\theta^{2}}\right)-\frac{\mathrm{a} U_{1}}{\theta^{2}}
$$


The formula thus becomes

$$
\begin{aligned}
\frac{4}{\Omega^{2}} \Delta A=\mathrm{aa}_{1}[M & +\left(\frac{2 U}{\theta^{3}}-\frac{U^{\prime}}{\theta^{2}}\right)\left(\partial \varpi+\mathrm{a}_{1} \partial u\right)^{2}+\left(-\frac{2 U_{1}}{\theta^{3}}-\frac{U_{1}^{\prime}}{\theta^{2}}\right)(\partial \varpi+\mathrm{a} \partial u)^{2} \\
& \left.-\left\{\frac{1}{\theta^{2}}\left(\operatorname{abcd}_{1} \mathrm{e}_{1} \mathrm{f}_{1}+\mathrm{a}_{1} \mathrm{~b}_{1} \mathrm{c}_{1} \mathrm{def}\right)-(\sqrt{d e})^{2}\right\}(\partial u)^{2}\right] \\
& -\frac{\mathrm{a}_{1} U}{\theta^{2}}\left(\partial \varpi+\mathrm{a}_{1} \partial u\right)^{2}-\frac{\mathrm{a} U_{1}}{\theta^{2}}(\partial \varpi+\mathrm{a} \partial u)^{2} \\
& +\left\{\frac{1}{\theta^{2}}\left(\operatorname{abcd}_{1} \mathrm{e}_{1} \mathrm{f}_{1}+\mathrm{a}_{1} \mathrm{~b}_{1} \mathrm{c}_{1} \mathrm{def}\right)-(\sqrt{d e})^{2}\right\}(\partial \varpi)^{2}
\end{aligned}
$$

viz. substituting for $M$ its value, the term in $(\sqrt{d e})^{2}(\partial u)^{2}$ disappears, and the formula is

$$
\begin{aligned}
& \frac{4}{\Omega^{2}} \Delta A=a a_{1}\left[\mathfrak{A}_{0}^{\prime}(\partial u)^{2}+2 \mathfrak{B}^{\prime} \partial u \partial \varpi+\delta^{\prime}(\partial \varpi)^{2}+\left(\frac{2 U}{\theta^{3}}-\frac{U^{\prime}}{\theta^{2}}\right)\left(\partial \varpi+\mathrm{a}_{1} \partial u\right)^{2}\right. \\
&\left.+\left(-\frac{2 U_{1}}{\theta^{3}}-\frac{U_{1}^{\prime}}{\theta^{2}}\right)(\partial \varpi+\mathrm{a} \partial u)^{2}-\frac{1}{\theta^{2}}\left(\operatorname{abcd}_{1} \mathrm{e}_{1} \mathrm{f}_{1}+\mathrm{a}_{1} \mathrm{~b}_{1} \mathrm{c}_{1} \mathrm{def}\right)(\partial u)^{2}\right] \\
&-\frac{\mathrm{a}_{1} U}{\theta^{2}}\left(\partial \varpi+\mathrm{a}_{1} \partial u\right)^{2}-\frac{\mathrm{a} U_{1}}{\theta^{2}}(\partial \varpi+\mathrm{a} \partial u)^{2} \\
&+\left\{\frac{1}{\theta^{2}}\left(\operatorname{abcd}_{1} \mathrm{e}_{1} \mathrm{f}_{1}+\mathrm{a}_{1} \mathrm{~b}_{1} \mathrm{c}_{1} \mathrm{def}\right)-(\sqrt{d e})^{2}\right\}(\partial \varpi)^{2}:
\end{aligned}
$$

say for shortness this is

$$
\begin{aligned}
\frac{4}{\Omega^{2}} & \Delta A \\
& =a a_{1} \Sigma-\frac{a_{1} U}{\theta^{2}}\left(\partial \varpi+a_{1} \partial u\right)^{2}-\frac{a U_{1}}{\theta^{2}}(\partial \varpi+a \partial u)^{2}+\frac{1}{\theta^{2}}\left(\operatorname{abcd}_{1} e_{1} f_{1}+a_{1} b_{1} c_{1} d e f\right)-(\sqrt{d e})^{2}(\partial \varpi)^{2} .
\end{aligned}
$$

Second step of the reduction.

In the reductions which follow, we make as many terms as may be to contain the factor $\mathrm{aa}_{1}$, so as to simplify as much as possible the portion not containing this factor.

We have $\partial \varpi+a_{1} \partial u=(\partial \varpi+\theta \partial u)+a \partial u$, and consequently

$$
\left(\partial \varpi+\mathrm{a}_{1} \partial u\right)^{2}=(\partial \varpi+\theta \partial u)^{2}+\mathrm{a} P,
$$

where $P=2 \partial u \partial \varpi+(a+2 \theta)(\partial u)^{2}$ : similarly $\partial \varpi+a \partial u=(\partial \varpi-\theta \partial u)+a_{1} \partial u$, and therefore

$$
(\partial \varpi+a \partial u)^{2}=(\partial \varpi-\theta \partial u)^{2}+a_{1} P_{1},
$$

where $P_{1}=2 \partial u \partial \sigma+\left(a_{1}-2 \theta\right)(\partial u)^{2}$ : the values may also be written

$$
P=2 \partial u \partial \varpi+\left(2 \mathrm{a}_{1}-\mathrm{a}\right)(\partial u)^{2}, \quad P_{1}=2 \partial u \partial \varpi+\left(2 \mathrm{a}-\mathrm{a}_{1}\right)(\partial u)^{2} .
$$


The formula thus becomes

$$
\begin{aligned}
\frac{4}{\Omega^{2}} \Delta A= & a_{1}\left\{\Sigma-\frac{U}{\theta^{2}} P-\frac{U_{1}}{\theta^{2}} P_{1}\right\} \\
& -\frac{a_{1} U}{\theta^{2}}(\partial \varpi+\theta \partial u)^{2}-\frac{a U_{1}}{\theta^{2}}(\partial \varpi-\theta \partial u)^{2}+\frac{1}{\theta^{2}}\left(\operatorname{abcd}_{1} \mathrm{e}_{1} \mathrm{f}_{1}+\mathrm{a}_{1} \mathrm{~b}_{1} \mathrm{c}_{1} \mathrm{def}\right)(\partial \varpi)^{2} \\
& -(\sqrt{d e})^{2}(\partial \varpi)^{2} .
\end{aligned}
$$

The second line here is

$$
\begin{aligned}
& -\left(a_{1} U+a U_{1}\right)(\partial u)^{2}-\frac{2}{\theta}\left(a_{1} U-a U_{1}\right) \partial u \partial \varpi \\
& +\frac{1}{\theta^{2}}\left\{-a_{1} U-a U_{1}+a b c d_{1} e_{1} f_{1}+a_{1} b_{1} c_{1} \operatorname{def}\right\}(\partial \varpi)^{2}
\end{aligned}
$$

and the coefficient herein of $(\partial \sigma)^{2}$ is $=\frac{1}{\theta^{2}}\left(\operatorname{ad}_{1} \mathrm{e}_{1} \mathrm{f}_{1}-a_{1} \mathrm{def}\right)\left(b c-b_{1} \mathrm{c}_{1}\right)$. Writing for the moment $d-a, e-a, f-a=d^{\prime}, e^{\prime}, f^{\prime}$, we have

$$
\begin{aligned}
\frac{1}{\theta}\left(a d_{1} e_{1} f_{1}-a_{1} d e f\right) & =\frac{1}{a_{1}-a}\left\{a\left(d^{\prime}+a_{1} \cdot e^{\prime}+a_{1} \cdot f^{\prime}+a_{1}\right)-a_{1}\left(d^{\prime}+a \cdot e^{\prime}+a \cdot f^{\prime}+a\right)\right\} \\
& =-d^{\prime} e^{\prime} f^{\prime}+a a_{1}\left(d^{\prime}+e^{\prime}+f^{\prime}+a+a_{1}\right), \\
\frac{1}{\theta}\left(b c-b_{1} c_{1}\right) & =-\left(b^{\prime}+c^{\prime}+a+a_{1}\right) .
\end{aligned}
$$

The whole term in $(\partial \varpi)^{2}$ is thus

$$
=\left\{\left(b^{\prime}+c^{\prime}+a+a_{1}\right) d^{\prime} e^{\prime} f^{\prime}+a_{1}\left(b^{\prime}+c^{\prime}+a+a_{1}\right)\left(d^{\prime}+e^{\prime}+f^{\prime}+a+a_{1}\right)\right\}(\partial \varpi)^{2} .
$$

The coefficient of $\partial u \partial \varpi$ is $-\frac{2}{\theta}\left(a_{1} U-a U_{1}\right)$ : viz. this is

$=\frac{2}{a_{1}-a}\left\{a_{1}\left(b^{\prime}+a \cdot c^{\prime}+a \cdot d^{\prime}+a \cdot e^{\prime}+a \cdot f^{\prime}+a\right)-a\left(b^{\prime}+a_{1} \cdot c^{\prime}+a_{1} \cdot d^{\prime}+a_{1} \cdot e^{\prime}+a_{1} \cdot f^{\prime}+a_{1}\right)\right\}$,

and if

that is,

this is

$$
b^{\prime}+a \cdot c^{\prime}+a \cdot d^{\prime}+a \cdot e^{\prime}+a \cdot f^{\prime}+a=B^{\prime}+C^{\prime} a+D^{\prime} a^{2}+E^{\prime} a^{3}+F^{\prime} a^{4}+a^{5}
$$

$$
\begin{gathered}
\mathbf{B}^{\prime}=b^{\prime} c^{\prime} d^{\prime} e^{\prime} f^{\prime}, \quad \mathbf{C}^{\prime}=\Sigma b^{\prime} c^{\prime} d^{\prime} e^{\prime}, \quad \mathbf{D}^{\prime}=\Sigma b^{\prime} c^{\prime} d^{\prime}, \quad \mathbf{E}^{\prime}=\Sigma b^{\prime} c^{\prime}, \\
\mathrm{F}^{\prime}=\Sigma b^{\prime}=b^{\prime}+c^{\prime}+d^{\prime}+e^{\prime}+f^{\prime},
\end{gathered}
$$

$$
=2\left\{B^{\prime}-D^{\prime} a a_{1}-E^{\prime} a a_{1}\left(a+a_{1}\right)-F^{\prime} a_{1} a_{1}\left(a^{2}+a a_{1}+a_{1}^{2}\right)-a a_{1}\left(a^{3}+a^{2} a_{1}+a a_{1}^{2}+a_{1}^{3}\right)\right\}:
$$

or say for shortness it is $=-2\left(B^{\prime}-a a_{1} \Phi\right)$ where

$$
\Phi=D^{\prime}+E^{\prime}\left(a+a_{1}\right)+F^{\prime}\left(a^{2}+a a_{1}+a_{1}^{2}\right)+a^{3}+a^{2} a_{1}+a a_{1}{ }^{2}+a_{1}^{3} ;
$$

the term in question thus is $-2\left(\mathrm{~B}^{\prime}-\mathrm{aa}_{1} \Phi\right) \partial u \partial \varpi$.

The coefficient of $(\partial u)^{2}$ is $-\left(a_{1} U+a U_{1}\right)$, viz. this is

$$
-a_{1}\left(b^{\prime}+a \cdot c^{\prime}+a \cdot d^{\prime}+a \cdot e^{\prime}+a \cdot f^{\prime}+a\right)-a\left(b^{\prime}+a_{1} \cdot c^{\prime}+a_{1} \cdot d^{\prime}+a_{1} \cdot e^{\prime}+a_{1} \cdot f^{\prime}+a_{1}\right),
$$

which is $=-\left(a+a_{1}\right) B^{\prime}-a a_{1} \Psi$, where

$$
\Psi=2 C^{\prime}+D^{\prime}\left(a+a_{1}\right)+E^{\prime}\left(a^{2}+a_{1}^{2}\right)+F^{\prime}\left(a^{3}+a_{1}^{3}\right)+a^{4}+a_{1}^{4} .
$$


The formula thus is

$$
\begin{aligned}
& \frac{4}{\Omega^{2}} \Delta A=\mathrm{aa}_{1}\left\{\Sigma-\frac{U}{\theta^{2}} P-\frac{U_{1}}{\theta^{2}} P_{1}-\left(b^{\prime}+c^{\prime}+\mathrm{a}+\mathrm{a}_{1}\right)\left(d^{\prime}+e^{\prime}+f^{\prime}+\mathrm{a}+\mathrm{a}_{1}\right)(\partial \varpi)^{2}+2 \Phi \partial u \partial \varpi-\Psi(\partial u)^{2}\right\} \\
&-\left(\mathrm{a}+\mathrm{a}_{1}\right) \mathrm{B}^{\prime}(\partial u)^{2}-2 \mathrm{~B}^{\prime} \partial u \partial \varpi+\left(b^{\prime}+c^{\prime}+\mathrm{a}+\mathrm{a}_{1}\right) d^{\prime} e^{\prime} f^{\prime}(\partial \varpi)^{2}-(\sqrt{d e})^{2}(\partial \varpi)^{2} .
\end{aligned}
$$

The whole coefficient of $a_{1}$, substituting for $\Sigma, P, P_{1}, \Phi, \Psi$ their values, and arranging according to $\partial \varpi, \partial u$, is

$$
\begin{aligned}
& =(\partial \varpi)^{2}\left\{\left(\delta^{\prime}+\frac{2 U}{\theta^{3}}-\frac{U^{\prime}}{\theta^{2}}+\left(-\frac{2 U_{1}}{\theta^{3}}-\frac{U_{1}^{\prime}}{\theta^{2}}\right)-\left(b^{\prime}+c^{\prime}+\mathrm{a}+\mathrm{a}_{1}\right)\left(d^{\prime}+e^{\prime}+f^{\prime}+\mathrm{a}+\mathrm{a}_{1}\right)\right\}\right. \\
& +2 \partial \varpi \partial u\left\{\mathfrak{B}^{\prime}+\mathrm{a}_{1}\left(\frac{2 U}{\theta^{3}}-\frac{U^{\prime}}{\theta^{2}}\right)+\mathrm{a}\left(-\frac{2 U_{1}}{\theta^{3}}-\frac{U_{1}^{\prime}}{\theta^{2}}\right)-\frac{U}{\theta^{2}}-\frac{U_{1}}{\theta^{2}}\right. \\
& \left.+D^{\prime}+E^{\prime}\left(a+a_{1}\right)+F^{\prime}\left(a^{2}+a a_{1}+a_{1}^{2}\right)+a^{3}+a^{2} a_{1}+a a_{1}{ }^{2}+a_{1}{ }^{3}\right\} \\
& +(\partial u)^{2}\left\{\mathfrak{A}_{0}{ }^{\prime}+\mathrm{a}_{1}{ }^{2}\left(\frac{2 U}{\theta^{3}}-\frac{U^{\prime}}{\theta^{2}}\right)+\mathrm{a}^{2}\left(-\frac{2 U_{1}}{\theta^{3}}-\frac{U_{1}{ }^{2}}{\theta^{2}}\right)+\left(\mathrm{a}-2 \mathrm{a}_{1}\right) \frac{U_{1}}{\theta^{2}}\right. \\
& \left.-\frac{1}{\theta^{2}}\left(a b c d_{1} e_{1} f_{1}+a_{1} b_{1} c_{1} d e f\right)-2 C^{\prime}-D^{\prime}\left(a+a_{1}\right)-E^{\prime}\left(a^{2}+a_{1}^{2}\right)-F^{\prime}\left(a^{3}+a_{1}^{3}\right)-a^{4}-a_{1}^{4}\right\}:
\end{aligned}
$$

and we have to reduce separately the three coefficients of this formula.

\section{Third step of the reduction.}

First, for the coefficient of $(\partial \varpi)^{2}$; recollecting that $\theta=a_{1}-a$, we have

$$
\begin{gathered}
2 \frac{U-U_{1}}{\theta}=-2 \mathrm{C}^{\prime}-2 \mathrm{D}^{\prime}\left(\mathrm{a}+\mathrm{a}_{1}\right)-2 \mathrm{E}^{\prime}\left(\mathrm{a}^{2}+\mathrm{aa}_{1}+\mathrm{a}_{1}^{2}\right)-2 \mathrm{~F}^{\prime}\left(\mathrm{a}^{3}+\mathrm{a}^{2} \mathrm{a}_{1}+a \mathrm{a}_{1}^{2}+\mathrm{a}_{1}^{3}\right) \\
-2\left(\mathrm{a}^{4}+\mathrm{a}^{3} \mathrm{a}_{1}+\mathrm{a}^{2} \mathrm{a}_{1}{ }^{2}+\mathrm{aa}_{1}^{3}+\mathrm{a}_{1}^{4}\right), \\
-\left(U^{\prime}+U_{1}^{\prime}\right)=2 \mathrm{C}^{\prime}+2 \mathrm{D}^{\prime}\left(\mathrm{a}+\mathrm{a}_{1}\right)+3 \mathrm{E}^{\prime}\left(\mathrm{a}^{2}+\mathrm{a}_{1}^{2}\right)+4 \mathrm{~F}^{\prime}\left(\mathrm{a}^{3}+\mathrm{a}_{1}^{3}\right)+5\left(\mathrm{a}^{4}+\mathrm{a}_{1}^{4}\right) .
\end{gathered}
$$

Adding these, the right-hand side divides by $\left(a_{1}-a\right)^{2}$, that is, by $\theta^{2}$; and the resulting value is

$$
=\mathrm{E}^{\prime}+2 \mathrm{~F}^{\prime}\left(\mathrm{a}+\mathrm{a}_{1}\right)+3 \mathrm{a}^{2}+4 \mathrm{aa}_{1}+3 \mathrm{a}_{1}^{2} .
$$

The term $-\left(b^{\prime}+c^{\prime}+a+a_{1}\right)\left(d^{\prime}+e^{\prime}+f^{\prime}+a+a_{1}\right)$, attending to the values of $\mathrm{E}^{\prime}$ and $\mathrm{F}^{\prime}$, is

$$
=b^{\prime} c^{\prime}+d^{\prime} e^{\prime}+d^{\prime} f^{\prime}+e^{\prime} f^{\prime}-\mathrm{E}^{\prime}-\mathrm{F}^{\prime}\left(\mathrm{a}+\mathrm{a}_{1}\right)-\mathrm{a}^{2}-2 a a_{1}-\mathrm{a}_{1}^{2}
$$

hence the whole coefficient of $(\partial \varpi)^{2}$ is

$$
=\left(5^{\prime}+b^{\prime} c^{\prime}+d^{\prime} e^{\prime}+d^{\prime} f^{\prime}+e^{\prime} f^{\prime}+\mathrm{F}^{\prime}\left(\mathrm{a}+\mathrm{a}_{1}\right)-2\left(\mathrm{a}^{2}+a \mathrm{a}_{1}+\mathrm{a}_{1}^{2}\right),\right.
$$

or substituting for $b^{\prime}, c^{\prime}, d^{\prime}, e^{\prime}, f^{\prime}$ their values, this is

$$
=c^{\prime}+4 a^{2}-a(b+c+2 d+2 e+2 f)+b c+d e+d f+e f+(\mathrm{F}-6 a)\left(\mathrm{a}+\mathrm{a}_{1}\right)-2\left(\mathrm{a}^{2}+\mathrm{aa}_{1}+\mathrm{a}_{1}^{2}\right) .
$$

Proceeding next to reduce the coefficient of $2 \partial \varpi \partial u$, observing as before that $\theta=a_{1}-a$, we have

$\frac{2 a_{1} U-2 a U_{1}}{\theta}=2 B^{\prime}-2 D^{\prime} a_{1}-2 E^{\prime} a_{1}\left(a+a_{1}\right)-2 F^{\prime} a a_{1}\left(a^{2}+a a_{1}+a_{1}^{2}\right)-2 a a_{1}\left(a^{3}+a^{2} a_{1}+a a_{1}^{2}+a_{1}^{3}\right)$,

C. $\mathrm{x}$. 
also

$$
\begin{aligned}
& -\left(\mathrm{a}_{1} U^{\prime}+U\right)-\left(\mathrm{a} U_{1}^{\prime}+U_{1}\right)= \\
& -2 B^{\prime}+D^{\prime}\left(-a^{2}+4 a a_{1}-a_{1}^{2}\right)+E^{\prime}\left(-a^{3}+3 a^{2} a_{1}+3 a a_{1}{ }^{2}-a_{1}{ }^{3}\right)+F^{\prime}\left(-a^{4}+4 a^{3} a_{1}+4 a a_{1}^{3}-a_{1}{ }^{4}\right) \\
& -a^{5}+5 a^{4} a_{1}+5 a a_{1}{ }^{4}-a_{1}{ }^{5}
\end{aligned}
$$

adding these two expressions, the right-hand side divides by $\left(a_{1}-a\right)^{2}$, that is, by $\theta^{2}$, and the resulting value is

$$
=-D^{\prime}-E^{\prime}\left(a+a_{1}\right)-F^{\prime}\left(a^{2}+a_{1}^{2}\right) \quad-a^{3}+a^{2} a_{1}+a a_{1}^{2}-a_{1}^{3} .
$$

To this is to be added

$$
+2 D^{\prime}+E^{\prime}\left(a+a_{1}\right)+F^{\prime}\left(a^{2}+a a_{1}+a_{1}^{2}\right)+a^{3}+a^{2} a_{1}+a a_{1}^{2}+a_{1}^{3}
$$

we thus see that the whole coefficient of $2 \partial u \partial \sigma$ is

or say it is

$$
=D^{\prime}+\quad F^{\prime} a a_{1}+2\left(a^{2} a_{1}+a a_{3}^{2}\right),
$$

$$
=D^{\prime}+(F-6 a) a a_{1}+2\left(a^{2} a_{1}+a a_{1}^{2}\right)
$$

Lastly, for the coefficient of $(\partial u)^{2}$; we have

$$
\frac{2 \mathrm{a}_{1}^{2} U-2 \mathrm{a}^{2} U_{1}}{\theta}=2 \mathrm{~B}^{\prime}\left(\mathrm{a}+\mathrm{a}_{1}\right)+2 \mathrm{C}^{\prime} \mathrm{aa}_{1}-2 \mathrm{E}^{\prime} \mathrm{a}^{2} \mathrm{a}_{1}{ }^{2}-2 \mathrm{~F}^{\prime} \mathrm{a}^{2} \mathrm{a}_{1}^{2}\left(\mathrm{a}+\mathrm{a}_{1}\right)-2 \mathrm{a}^{2} \mathrm{a}_{1}^{2}\left(\mathrm{a}^{2}+\mathrm{aa}_{1}+\mathrm{a}_{1}^{2}\right)
$$

and also

$$
\begin{aligned}
-a_{1}^{2} U^{\prime} & +\left(a-2 a_{1}\right) U-a^{2} U_{1}^{\prime}+\left(a_{1}-2 a\right) U= \\
& -B^{\prime}\left(a+a_{1}\right)+C^{\prime}\left(2 a^{2}-4 a a_{1}+2 a_{1}^{2}\right)+D^{\prime}\left(a^{3}+a_{1}^{3}\right)+E^{\prime}\left(a^{4}-2 a^{3} a_{1}+6 a^{2} a_{1}^{2}-2 a a_{1}^{3}+a_{1}^{4}\right) \\
& +F^{\prime}\left(a^{5}-2 a^{4} a_{1}+4 a^{3} a_{1}^{2}+4 a^{2} a_{1}^{3}-2 a a_{1}^{4}+a_{1}^{5}\right)+\left(a^{6}-2 a^{5} a_{1}+5 a^{4} a_{1}{ }^{2}+5 a^{2} a_{1}{ }^{4}-2 a a_{1}{ }^{5}+a_{1}^{6}\right)
\end{aligned}
$$

whence the sum of these two expressions is

$$
\begin{aligned}
=B^{\prime} & \left(a+a_{1}\right)+C^{\prime}\left(2 a^{2}-2 a a_{1}+2 a_{1}^{2}\right)+D^{\prime}\left(a^{3}+a_{1}^{3}\right)+E^{\prime}\left(a^{4}-2 a^{3} a_{1}+4 a^{2} a_{1}^{2}-2 a a_{1}^{3}+a_{1}^{4}\right) \\
& +F^{\prime}\left(a^{5}-2 a^{4} a_{1}+2 a^{3} a_{1}{ }^{2}+2 a^{2} a_{1}^{3}-2 a a_{1}{ }^{4}+a_{1}^{5}\right)+a^{6}-2 a^{5} a_{1}+3 a^{4} a_{1}{ }^{2}-2 a^{3} a_{1}{ }^{3}+3 a^{2} a_{1}^{4}-2 a a_{1}{ }^{5}+a_{1}{ }^{6} .
\end{aligned}
$$

We must to this add the term $-\left(\operatorname{abcd}_{1} \mathrm{e}_{1} \mathrm{f}_{1}+\mathrm{a}_{1} \mathrm{~b}_{1} \mathrm{c}_{1} \mathrm{def}\right)$, that is,

$$
-\mathrm{a}\left(b^{\prime}+\mathrm{a} \cdot c^{\prime}+\mathrm{a} \cdot d^{\prime}+\mathrm{a}_{1} \cdot e^{\prime}+\mathrm{a}_{1} \cdot f^{\prime}+\mathrm{a}_{1}\right)-\mathrm{a}_{1}\left(b^{\prime}+\mathrm{a}_{1} \cdot c^{\prime}+\mathrm{a}_{1} \cdot d^{\prime}+\mathrm{a} \cdot e^{\prime}+\mathrm{a} \cdot f^{\prime}+\mathrm{a}\right) \text {. }
$$

Putting for the moment

that is,

$$
d^{\prime}+\mathrm{a} \cdot e^{\prime}+\mathrm{a} \cdot f^{\prime}+\mathrm{a}=\tau^{\prime}+\sigma^{\prime} \mathrm{a}+\rho^{\prime} \mathrm{a}^{2}+\mathrm{a}^{3},
$$

the term is

$$
\tau^{\prime}=d^{\prime} e^{\prime} f^{\prime}, \quad \sigma^{\prime}=d^{\prime} e^{\prime}+d^{\prime} f^{\prime}+e^{\prime} f^{\prime}, \quad \rho^{\prime}=d^{\prime}+e^{\prime}+f^{\prime},
$$

$$
\begin{aligned}
-b^{\prime} c^{\prime} \tau^{\prime}\left(\mathrm{a}+\mathrm{a}_{1}\right)- & \left(b^{\prime}+c^{\prime}\right) \tau^{\prime}\left(\mathrm{a}^{2}+\mathrm{a}_{1}{ }^{2}\right)-\tau^{\prime}\left(\mathrm{a}^{3}+\mathrm{a}_{1}{ }^{3}\right)-\left(b^{\prime} c^{\prime}+\sigma^{\prime}\right)\left(\mathrm{a}^{3} \mathrm{a}_{1}+\mathrm{aa}_{1}{ }^{3}\right) \\
- & \left(b^{\prime}+c^{\prime}+\rho^{\prime}\right)\left(\mathrm{a}^{3} \mathrm{a}_{1}{ }^{2}+\mathrm{a}^{2} \mathrm{a}_{1}{ }^{3}\right)-2 \mathrm{a}^{3} \mathrm{a}_{1}{ }^{3} \\
& -2 b^{\prime} c^{\prime} \sigma^{\prime} \mathrm{aa}_{1}-\left[\left(b^{\prime}+c^{\prime}\right) \sigma^{\prime}+b^{\prime} c^{\prime} \rho^{\prime}\right]\left(\mathrm{a}^{2} \mathrm{a}_{1}+\mathrm{aa}_{1}{ }^{2}\right)-2\left(b^{\prime}+c^{\prime}\right) \rho^{\prime} \mathrm{a}^{2} \mathrm{a}_{1}{ }^{2} .
\end{aligned}
$$


Adding it to the preceding expression, the sum is

$$
\begin{aligned}
& =\left(\mathrm{B}^{\prime}-b^{\prime} c^{\prime} \tau^{\prime}\right)\left(\mathrm{a}+\mathrm{a}_{1}\right)+\left\{2 \mathrm{c}^{\prime}-\left(b^{\prime}+c^{\prime}\right) \tau^{\prime}\right\}\left(\mathrm{a}^{2}+\mathrm{a}_{1}^{2}\right)+\left(\mathrm{D}^{\prime}-\tau^{\prime}\right)\left(\mathrm{a}^{3}+\mathrm{a}_{1}{ }^{3}\right)+\mathrm{E}^{\prime}\left(\mathrm{a}^{4}+\mathrm{a}_{1}^{4}\right) \\
& +\left\{-2 c^{\prime}-2 b^{\prime} c^{\prime} \sigma^{\prime}\right\} a a_{1}-\left\{\left(b^{\prime}+c^{\prime}\right) \sigma^{\prime}+b^{\prime} c^{\prime} \rho^{\prime}\right\}\left(a^{2} a_{1}+a a_{1}^{2}\right)-\left(2 \mathrm{E}^{\prime}+b^{\prime} c^{\prime}+\sigma^{\prime}\right)\left(a^{3} a_{1}+a a_{1}^{3}\right) \\
& +\left\{4 \mathrm{E}^{\prime}-2\left(b^{\prime}+c^{\prime}\right) \rho^{\prime}\right\} \mathrm{a}^{2} \mathrm{a}_{1}{ }^{2} \\
& +\mathrm{F}^{\prime}\left(\mathrm{a}^{5}+\mathrm{a}_{1}^{5}\right) \quad+\mathrm{a}^{6}+\mathrm{a}_{1}{ }^{6} \\
& -2 \mathrm{~F}^{\prime}\left(\mathrm{a}^{4} \mathrm{a}_{1}+\mathrm{aa}_{1}^{4}\right) \quad-2\left(\mathrm{a}^{5} \mathrm{a}_{1}+\mathrm{aa}_{1}^{5}\right) \\
& +\left(2 \mathrm{~F}^{\prime}-b^{\prime}-c^{\prime}-\rho^{\prime}\right)\left(\mathrm{a}^{3} \mathrm{a}_{1}{ }^{2}+\mathrm{a}^{2} \mathrm{a}_{1}^{3}\right)+3\left(\mathrm{a}^{4} \mathrm{a}_{1}{ }^{2}+\mathrm{a}^{2} \mathrm{a}_{1}{ }^{4}\right)-4 \mathrm{a}^{3} \mathrm{a}_{1}^{3} \text {. }
\end{aligned}
$$

This is, in fact, divisible by $\left(a_{1}-a\right)^{2}$, that is, by $\theta^{2}$ : for we have between the symbols the relations

$$
\begin{aligned}
& \mathrm{F}^{\prime}=b^{\prime}+c^{\prime}+\rho^{\prime}, \\
& \mathrm{E}^{\prime}=b^{\prime} c^{\prime}+\left(b^{\prime}+c^{\prime}\right) \rho^{\prime}+\sigma^{\prime}, \\
& \mathrm{D}^{\prime}=b^{\prime} c^{\prime} \rho^{\prime}+\left(b^{\prime}+c^{\prime}\right) \sigma^{\prime}+\tau^{\prime}, \\
& \mathrm{C}^{\prime}=b^{\prime} c^{\prime} \sigma^{\prime}+\left(b^{\prime}+c^{\prime}\right) \tau^{\prime}, \\
& \mathrm{B}^{\prime}=b^{\prime} c^{\prime} \tau^{\prime}
\end{aligned}
$$

and we thus reduce the expression to

$$
\begin{aligned}
\left\{2 \mathrm{c}^{\prime}-\left(b^{\prime}\right.\right. & \left.\left.+c^{\prime}\right) \tau^{\prime}\right\}\left(\mathrm{a}^{2}-2 \mathrm{aa}_{1}+\mathrm{a}_{1}{ }^{2}\right)+\left(\mathrm{D}^{\prime}-\tau^{\prime}\right)\left(\mathrm{a}^{3}-\mathrm{a}^{2} \mathrm{a}_{1}-\mathrm{aa}_{1}{ }^{2}+\mathrm{a}_{1}{ }^{3}\right) \\
& +\mathrm{E}^{\prime}\left(\mathrm{a}^{4}-3 \mathrm{a}^{3} \mathrm{a}_{1}+4 \mathrm{a}^{2} \mathrm{a}_{1}{ }^{2}-3 \mathrm{aa}_{1}{ }^{3}+\mathrm{a}_{1}^{4}\right)+\left(b^{\prime}+c^{\prime}\right) \rho^{\prime}\left(\mathrm{a}^{3} \mathrm{a}_{1}-2 \mathrm{a}^{2} \mathrm{a}_{1}{ }^{2}+\mathrm{aa}_{1}{ }^{3}\right) \\
& +\mathrm{F}^{\prime}\left(\mathrm{a}^{5}-2 \mathrm{a}^{4} \mathrm{a}_{1}+\mathrm{a}^{3} \mathrm{a}_{1}{ }^{2}+\mathrm{a}^{2} \mathrm{a}_{1}{ }^{3}-2 \mathrm{aa}_{1}{ }^{4}+\mathrm{a}_{1}{ }^{5}\right) \\
& +\left(\mathrm{a}^{6}-2 \mathrm{a}^{5} \mathrm{a}_{1}+3 \mathrm{a}^{4} \mathrm{a}_{1}{ }^{2}-4 \mathrm{a}^{3} \mathrm{a}_{1}{ }^{3}+3 \mathrm{a}^{2} \mathrm{a}_{1}{ }^{4}-2 \mathrm{aa}_{1}{ }^{5}+\mathrm{a}_{1}{ }^{6}\right),
\end{aligned}
$$

viz. effecting the division, the quotient is

$=2 \mathrm{C}^{\prime}-\left(b^{\prime}+c^{\prime}\right) \tau^{\prime}+\left(\mathrm{D}^{\prime}-\tau^{\prime}\right)\left(\mathrm{a}+\mathrm{a}_{1}\right)+\mathrm{E}^{\prime}\left(\mathrm{a}^{2}+\mathrm{a}_{1}{ }^{2}\right)+\mathrm{F}^{\prime}\left(\mathrm{a}^{3}+\mathrm{a}_{1}^{3}\right)+\mathrm{a}^{4}+2 \mathrm{a}^{2} \mathrm{a}_{1}{ }^{2}+\mathrm{a}_{1}{ }^{4}-\left(b^{\prime} c^{\prime}+\sigma^{\prime}\right) a \mathrm{a}_{1}$.

To this must be added

$-2 c^{\prime}$

$$
-D^{\prime}\left(a+a_{1}\right) \quad-E^{\prime}\left(a^{2}+a_{1}^{2}\right)-F^{\prime}\left(a^{3}+a_{1}^{3}\right)-\left(a^{4}+a_{1}^{4}\right) ;
$$

and we thus obtain the coefficient of $(\partial u)^{2}$ in the form

$$
\mathfrak{A}_{0}^{\prime}-\left(b^{\prime}+c^{\prime}\right) \tau^{\prime}-\tau^{\prime}\left(\mathrm{a}+\mathrm{a}_{1}\right)-\left(b^{\prime} c^{\prime}+\sigma^{\prime}\right) \mathrm{aa}_{1}+2 \mathrm{a}^{2} \mathrm{a}_{1}{ }^{2},
$$

viz. this is

$$
\begin{aligned}
= & \mathfrak{A}_{0}^{\prime}+(b+c-2 a)(a-d)(a-e)(a-f)+(a-d)(a-e)(a-f)\left(a+a_{1}\right) \\
& +\{-(a-b)(a-c)-(a-d)(a-e)-(a-d)(a-f)-(a-e)(a-f)\} a_{1}+2 a^{2} a_{1}{ }^{2} \\
& 27-2
\end{aligned}
$$


or finally it is

$$
\begin{aligned}
=\mathfrak{A}_{0}^{\prime}-2 a^{4} & +a^{3}(b+c+2 d+2 e+2 f)+a^{2}\{-(b+c)(d+e+f)-2(d e+d f+e f)\} \\
& +a\{(b+c)(d e+d f+e f)+2 d e f\}-(b+c) d e f \\
& +\left\{a^{3}-a^{2}(d+e+f)+a(d e+d f+e f)-d e f\right\}\left(a+a_{1}\right) \\
& +\left\{-4 a^{2}+a(b+c+2 d+2 e+2 f)-b c-d e-d f-e f\right\} a_{1} \\
& +2 a^{2} a_{1}^{2} .
\end{aligned}
$$

It is to be observed that the investigation thus far has been entirely independent of the values of $\mathfrak{A}_{0}^{\prime}, \mathfrak{B}^{\prime}, \mathfrak{C}^{\prime}$ : these values are, in fact, such as to make the coefficients of $(\partial \varpi)^{2}, \partial \varpi \partial u,(\partial u)^{2}$ each equal to a constant, and it was really by such a condition that the value of $\widetilde{C}\left(=\widetilde{C}^{\prime}\right)$ was determined; but if we had thus also determined the values of $\mathfrak{A}_{0}^{\prime}$ and $\mathfrak{B}^{\prime}$, it would not have been apparent that the values of $\mathfrak{A}_{0}^{\prime}, \mathfrak{B}^{\prime}$ and ${ }^{\prime} \mathcal{S}^{\prime}$ thus determined would be consistent with each other: the foregoing investigation of these values was therefore prefixed.

Completion of the reduction and final expression for $\Delta A$.

But now substituting the values of $\mathfrak{A}_{0}^{\prime}, \mathfrak{B}^{\prime}$, $\mathfrak{C}^{\prime}$, we find

$$
\begin{aligned}
\text { coeff. of } \quad(\partial \varpi)^{2}= & a b+a c+b c+d e+d f+e f \\
\# \quad \Rightarrow \quad 2 \partial \varpi \partial u= & -a^{2}(a-b-c-d-e-f) \\
\Rightarrow \quad \Rightarrow \quad(\partial u)^{2}= & -2 a^{4}+2 a^{3}(b+c+d+e+f) \\
& -a^{2}(b c+b d+b e+b f+c d+c e+c f+d e+d f+e f) \\
& -(b c d e+b c d f+b c e f+b d e f+c d e f),
\end{aligned}
$$

viz. these coefficients belong to the portion which contains the factor $a a_{1}$ of the expression for $\frac{4}{\Omega^{2}} \Delta A:$ the other portion was

where

$$
\left(b^{\prime}+c^{\prime}+a+a_{1}\right) d^{\prime} e^{\prime} f^{\prime}(\partial \varpi)^{2}-(\sqrt{d e})^{2}(\partial \varpi)^{2}-2 B^{\prime} \partial u \partial \varpi-\left(a+a_{1}\right) B^{\prime}(\partial u)^{2},
$$

$$
\mathrm{B}^{\prime}=b^{\prime} c^{\prime} d^{\prime} e^{\prime} f^{\prime}, \quad b^{\prime}=b-a, \text { etc. }
$$

We have thus the complete result, viz. this is

$$
\begin{aligned}
\frac{4}{\Omega^{2}} \Delta A= & a_{1}\left[(a b+a c+b c+d e+d f+e f)(\partial \varpi)^{2}\right. \\
& -a^{2}(a-b-c-d-e-f) 2 \partial \varpi \partial u \\
& +\left\{\begin{array}{l}
-2 a^{4}+2 a^{3}(b+c+d+e+f) \\
\left.\left.-a^{2}(b c+b d+b e+b f+c d+c e+c f+d e+d f+e f)\right\}(\partial u)^{2}\right] \\
-(b c d e+b c d f+b c e f+b d e f+c d e f)
\end{array}\right\} \\
& -\left(-2 a+b+c+a+a_{1}\right)(a-d)(a-e)(a-f)(\partial \varpi)^{2}-(\sqrt{d e})^{2}(\partial \varpi)^{2} \\
& +(a-b)(a-c)(a-d)(a-e)(a-f) 2 \partial u \partial \varpi \\
& +\left(a+a_{1}\right)(a-b)(a-c)(a-d)(a-e)(a-f)(\partial u)^{2}
\end{aligned}
$$

which is obviously a sum of squares. 
As a partial verification, I remark that $\Delta A$ should be symmetrical in regard to the constants $b, c, d, e, f$; this is obviously the case as regards the terms in $\partial u \partial \sigma$ and $(\partial u)^{2}$, and it must also be so in regard to the term in $(\partial \varpi)^{2}$. The whole coefficient of $(\partial \sigma)^{2}$ is

$$
\begin{aligned}
= & a a_{1}(a b+a c+b c+d e+d f+e f) \\
& -\left(-2 a+b+c+a+a_{1}\right)(a-d)(a-e)(a-f)-(\sqrt{d e})^{2},
\end{aligned}
$$

and if we interchange for instance $b$ and $d$, this coefficient becomes

$$
\begin{aligned}
= & a a_{1}(a d+a c+c d+b e+b f+e f) \\
& -\left(-2 a+d+c+a+a_{1}\right)(a-b)(a-e)(a-f)-(\sqrt{b e})^{2} .
\end{aligned}
$$

These two expressions must be equal; viz. we must have

$$
(\sqrt{b e})^{2}-(\sqrt{d e})^{2}=-a a_{1}(b-d)(a+c-e-f)+(a-e)(a-f)(b-d)\left(-a+c+a+a_{1}\right):
$$

the left-hand side is

$$
=\frac{1}{\theta^{2}}\left(b d_{1}-b_{1} d\right)\left(e a_{1} c_{1}-e_{1} f_{1} a c\right)
$$

and we have

$$
\mathrm{bd}_{1}-\mathrm{b}_{1} \mathrm{~d}=(b-d) \theta
$$

hence, throwing out the factor $b-d$, the equation to be verified becomes

$$
\frac{1}{\theta}\left(\mathrm{efa}_{1} \mathrm{c}_{1}-\mathrm{e}_{1} \mathrm{f}_{1} \mathrm{ac}\right)=-\mathrm{aa}_{1}(a+c-e-f)+(a-e)(a-f)\left(-a+c+\mathrm{a}+\mathrm{a}_{1}\right)
$$

Writing

the left-hand side is

$$
\text { e }=e^{\prime}+\mathrm{a} \text {, etc., } \quad \theta=\mathrm{a}_{1}-\mathrm{a},
$$

$$
\left(a+a_{1}\right) e^{\prime} f^{\prime}+a a_{1}\left(e^{\prime}+f^{\prime}\right)+c^{\prime} e^{\prime} f^{\prime}-c^{\prime} a_{1}
$$

and the right-hand side is

and these are equal.

$$
-a a_{1}\left(c^{\prime}-e^{\prime}-f^{\prime}\right)+e^{\prime} f^{\prime}\left(c^{\prime}+a+a_{1}\right)
$$

There are of course, in all, six expressions such as $\Delta A$, each of them being by what precedes a sum of squares. And there are besides ten expressions such as

$$
\Delta A B,=A B \partial^{2} A B-(\partial A B)^{2},
$$

each of which should be a sum of squares: but $I$ have not as yet effected the calculation of this expression $\triangle A B$.

Cambridge, 7th December, 1877. 